\title{
Lepton-flavor violating mediators
}

\author{
Iftah Galon, ${ }^{a}$ Anna Kwa ${ }^{a}$ and Philip Tanedo ${ }^{b}$ \\ ${ }^{a}$ Department of Physics $\&$ Astronomy, University of California, \\ Irvine, CA 92697, U.S.A. \\ ${ }^{b}$ Department of Physics $\&$ Astronomy, University of California, \\ Riverside, CA 92521, U.S.A. \\ E-mail: iftachg@uci.edu, akwa@uci.edu, flip.tanedo@ucr.edu
}

ABSTRACT: We present a framework where dark matter interacts with the Standard Model through a light, spin-0 mediator that couples chirally to pairs of different-flavor leptons. This flavor violating final state weakens bounds on new physics coupled to leptons from terrestrial experiments and cosmic-ray measurements. As an example, we apply this framework to construct a model for the Fermi-LAT excess of $\mathrm{GeV} \gamma$-rays from the galactic center. We comment on the viability of this portal for self-interacting dark matter explanations of small scale structure anomalies and embeddings in flavor models. Models of this type are shown to be compatible with the muon anomalous magnetic moment anomaly. We review current experimental constraints and identify possible future theoretical and experimental directions.

Keywords: Supersymmetry Phenomenology

ARXIV EPRINT: 1610.08060 


\section{Contents}

1 Introduction 1

2 Models of a lepton-flavor violating mediator 4

2.1 Dark sector interactions 4

2.2 Standard model interactions 6

2.3 Comments on ultraviolet realizations $\quad 6$

$\begin{array}{lll}2.4 & \text { Self-interacting dark matter } & 7\end{array}$

3 The Fermi-LAT $\gamma$-ray excess $\quad 8$

3.1 Photons from leptons 8

$3.2 \quad$ Fit to Fermi $\gamma$-ray excess 9

4 The AMS-02 $e^{ \pm}$spectrum $\quad 14$

5 Constraints on standard model couplings $\quad 16$

$\begin{array}{lll}5.1 & \text { Photon lines } & 16\end{array}$

$\begin{array}{lll}5.2 & \text { Electroweak precision measurements } & 16\end{array}$

$\begin{array}{lll}5.3 & \text { Lepton anomalous dipole moments } & 17\end{array}$

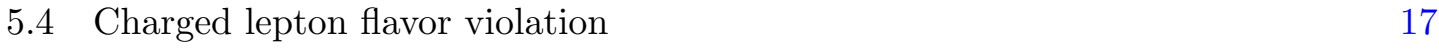

$\begin{array}{ll}5.5 \text { Direct detection } & 19\end{array}$

5.6 Dark photon searches 19

$\begin{array}{lll}5.7 & \text { Collider searches } & 20\end{array}$

6 Conclusions \& outlook 20

\section{Introduction}

Scenarios where dark matter is a thermal relic that interacts directly with the Standard Model are typically constrained by a range of complementary experimental searches [1]. On the other hand, if dark matter is secluded from the Standard Model and only interacts through a light mediator, then one may obtain the observed relic density from thermal freeze out while parametrically suppressing signatures in direct detection and collider experiments [2]. Direct annihilation into on-shell mediators sets the dark matter-mediator couplings, while the mediator-Standard Model couplings can be small enough to explain the null results from direct detection and collider experiments. Dark matter continues to annihilate in the present day and the Standard Model byproducts of the ensuing mediator decays may be observable. 
One possible signature consistent with this framework is the recent excess of $\gamma$-rays from the Galactic Center observed by independent analyses of the Fermi Large Area Telescope (LAT) data [3-19] and directly by the Fermi-LAT collaboration [20]. Alternative explanations include unresolved pulsars [5, 8, 14, 21-27] or cosmic ray outbursts [28-31]. The excess can be fit to effective theories that describe the annihilation of dark matter into pairs of Standard Model particles [32-37]. Intriguingly, the total flux of excess $\gamma$-rays is consistent with the $s$-wave dark matter annihilation cross-section required to produce the observed relic density after thermal freeze out. Early fits to the energy spectrum preferred $40 \mathrm{GeV}$ dark matter annihilating to $b$-quarks or $10 \mathrm{GeV}$ dark matter annihilating into $\tau$-leptons; however, later studies found that masses up to $\mathcal{O}(100 \mathrm{GeV})$ and a range of final states are allowed after accounting for the systematic uncertainties in the astrophysical background models [16-20,37]. When dark matter annihilates into on-shell mediators in the secluded dark matter framework, the target space is shifted towards heavier dark matter and larger annihilation cross-sections [38-42].

Most proposals to explain the excess from dark matter annihilations focus on $\gamma$-ray emission from bremsstrahlung and $\pi^{0}$ decays of annihilation products. These processes produce prompt $\gamma$-rays at the site of annihilation with intensities directly proportional to the square of the dark matter density. This predicts a similar signal in dwarf spheroidal galaxies which are rich in dark matter and have simpler astrophysical backgrounds than the Galactic Center [43]. The non-observation of a clear excess in dwarf spheroidal galaxies [44, $45]$ is typically a tension in dark matter interpretations of the $\gamma$-ray excess, indications of possible excesses in Reticulum II [46, 47] and Tucana III [48] notwithstanding.

Lacroix, Boehm, and Silk pointed out that another mechanism by which the Galactic Center excess might be generated is through the inverse Compton scattering (ICS) of finalstate electrons and positrons with infrared starlight [49]. The energetic $e^{+} e^{-}$pairs upscatter the low-energy photons into the GeV range. Recently, Calore et al. and Kaplinghat et al. proposed the possibility that these electron pairs may result from the decay of on-shell mediators $[18,50]$. Up-scattering of starlight effectively does not occur in dwarf galaxies because of their much weaker interstellar radiation field. This removes the tension between the Fermi Galactic Center and dwarf $\gamma$-ray observations. In its simplest form, however, this scenario is in tension with a different astrophysical observation. Direct dark matter annihilation into $e^{+} e^{-}$pairs produces a line in the local $e^{+} e^{-}$spectrum that is observable by the Alpha Magnetic Spectrometer (AMS-02) telescope. The absence of such a line requires a mechanism to soften the primary $e^{+} e^{-}$spectrum. Kaplinghat, Linden, and Yu realize this in the secluded dark matter scenario in which the annihilations into light mediators broadens the spectrum of daughter electrons and positrons. When there is a hierarchy in the dark matter $\chi$ and mediator $\varphi$ masses, $m_{\chi} \gg m_{\varphi} \gg m_{e}$, the production spectrum of electrons is smeared from a line at $m_{\chi}$ to a box from 0 to $m_{\chi}$. In this way, one may attempt to hide the electron-positron spectrum by smearing it out within the AMS-02 error bars. The benchmark model in [50] invokes $50 \mathrm{GeV}$ dark matter annihilating into $100 \mathrm{MeV}$ spin-1 mediators. As an additional feature, these masses automatically furnish the ingredients for a self-interacting dark matter solution to outstanding small-scale structure anomalies as reviewed in $[51,52]$. The resulting dark matter annihilation cross-section is smaller than 
that of a thermal relic in the visible sector and thus requires the dark sector temperature to differ from the Standard Model at freeze out.

In this manuscript we introduce an alternative class of models that produce a $\gamma$-ray signal in the galactic center while avoiding bounds from the non-observation of such a signal in dwarf galaxies. Like Calore et al. and Kaplinghat et al., dark matter annihilates into on-shell mediators and the $\gamma$-ray signal is produced from the up-scattering of starlight. In our case, however, the mediator is a spin-0 particle which decays into different-flavor lepton pairs, $\tau \mu, \tau e$ or $\mu e$. The final $e^{ \pm}$spectrum is softer which allows it to better fit within the error bars of the AMS-02 observations. In the models with $\tau$ couplings, the hadronic $\tau$ decays yield prompt photons which contribute to the $\gamma$-ray excess in the absence of an interstellar radiation field. This, in turn, re-introduces tension with the dwarfs. As observed in [39, 41], the leading $s$-wave contribution to dark matter annihilation into pseudoscalars is the $2 \rightarrow 3$ process that further softens the spectrum of ensuing the Standard Model decay products. We show that the resulting $\gamma$-ray spectra are consistent with the Fermi-LAT excess for an annihilation cross-section that is compatible with the correct dark matter relic density even when the dark sector and Standard Model have the same temperature at freeze out. In the case where the mediator also contains a parity-even spin-0 component, this framework may still furnish a self-interacting dark matter solution to small-scale structure anomalies.

New sources of lepton flavor violation are strongly constrained by a plethora of flavor observables. Though we include a flavor-violating coupling, our scenario avoids many of these constraints by preserving a residual $L_{i}-L_{j}$ global Abelian symmetry, under which the mediator has charge 2. This restricts mediator-SM interactions to: (a) a chiral coupling to a single oppositely-charged, different-flavored lepton pair, and (b) scalar potential terms proportional to powers of $\varphi^{*} \varphi$. The symmetry prohibits most charged lepton flavorviolating processes while the chiral structure suppresses contributions to flavor-diagonal observables such as anomalous dipole moments. The leading constraints on the mediator's leptonic coupling come from the forward-backward asymmetries in electron collisions and from the anomalous magnetic moment of the muon. We identify possible future experimental directions in dark photon and collider searches.

The proposed coupling structure can naturally arise at the electroweak scale if a theory of flavor is responsible for the structure of both the Standard Model and mediator leptonic couplings. One example is to use the Froggatt-Nielsen mechanism [53] and break its flavor symmetry at $\sim \mathrm{TeV}$. While this coupling structure is not stable under renormalization group evolution, loop corrections cannot substantially reintroduce additional flavor combinations of mediator leptonic couplings. This phenomenon is due to the $L_{i}-L_{j}$ symmetry breaking spurions being proportional to the neutrino masses and the small electroweakscale $\varphi$ couplings.

Recently, light, lepton flavor violating bosons have been a topic of interest [54, 55]. Part of the interest has been driven by a possible breakdown of lepton flavor universality in $B$-meson decays [56-60] and early suggestions of the observation of the lepton-flavor violating Higgs decay, $h \rightarrow \mu \tau[61,62]$. We point out that independent of the connection to possible astrophysical signals, the features of the lepton flavor violating interactions here 


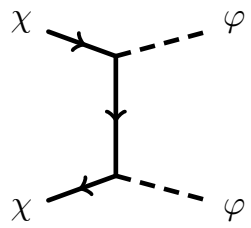

(a) $2 \chi \rightarrow 2 \varphi$

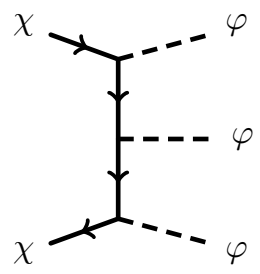

(b) $2 \chi \rightarrow 3 \varphi$

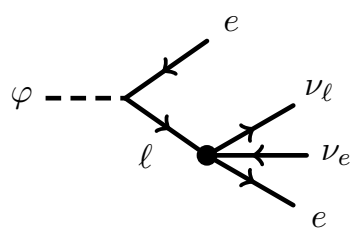

(c) el decay

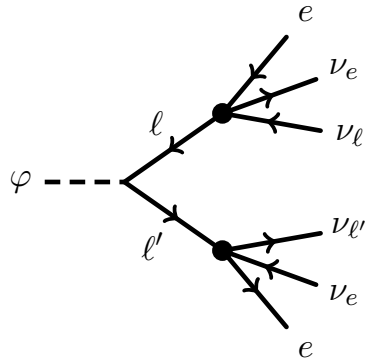

(d) $\ell \ell^{\prime}$ decay

Figure 1. Diagrams showing annihilation to mediators and possible off-diagonal mediator decay modes. The dot is a Fermi vertex from an off-shell $W$ for the subsequent decay of heavy leptons. $\tau$ final states also carry a large hadronic branching ratio.

are an interesting example for light, weakly coupled new physics which may have non-trivial signatures while evading existing bounds. In addition, while our models are benchmarked against the Fermi-LAT $\gamma$-ray excess, they constitute a new class of simplified dark matter models where the mediator interacts with the Standard Model through chiral, lepton-flavor violating couplings. This fits into a larger body of literature studying dark sectors with non-trivial properties under the Standard Model flavor symmetries [63-81].

\section{Models of a lepton-flavor violating mediator}

We present our model in this section; the interactions are summarized in figure 1.

\subsection{Dark sector interactions}

Fermionic dark matter $\chi$ is proposed to interact with a spin-0 mediator, $\varphi$, through the interactions,

$$
\mathcal{L}_{\varphi} \chi=\frac{1}{2} y_{S} \eta \varphi \bar{\chi} \chi+\frac{i}{2} y_{P} \eta \varphi \bar{\chi} \gamma^{5} \chi \quad \eta=1(2) \text { for Majorana (Dirac) } \chi .
$$

If $\varphi$ is complex, one must also include $\mathcal{L}_{\varphi \chi}^{\dagger}$. Unlike the case of a vector mediator for which there is no coupling to Majorana dark matter, the spin-0 mediator can couple to either Dirac or Majorana fermions. In writing these interactions, we assume that the fermion mass is manifestly real so that the pseudoscalar interaction is physical. See, for example, ref. [82] for a demonstration of how a complex fermion mass term - say, if $\langle\varphi\rangle$ and $y_{P} \neq 0$ - would shift the pseudoscalar interaction upon performing a chiral rotation to make the mass term real. For the remainder of this manuscript we assume that $\langle\varphi\rangle=0$.

In the secluded dark matter scenario where annihilation goes into on-shell mediators, the dark matter-mediator couplings of (2.1) control the annihilation rate independently of the mediator-Standard Model couplings. A useful benchmark is the annihilation crosssection required for $\chi$ to reproduce the observed dark matter density assuming that it was initially in thermal equilibrium with the Standard Model [83],

$$
\langle\sigma v\rangle_{\mathrm{rel} .}=2.2 \eta \times 10^{-26} \mathrm{~cm}^{3} / \mathrm{s} .
$$


Below we present targets for the dark matter-mediator couplings implicitly as a ratio of the required present day annihilation cross-section, $\langle\sigma v\rangle$ set by the Fermi $\gamma$-ray excess, to the thermal relic cross-section, $\langle\sigma v\rangle_{\text {rel. }}$. In doing so, we cancel all dependence on $\eta$ in our results. The extent to which $\langle\sigma v\rangle$ is compatible with $\langle\sigma v\rangle_{\text {rel. }}$ is a useful metric of attractiveness for our models. Compatibility is simplest when annihilation is dominantly $s$-wave since higher partial waves are highly velocity suppressed in the present day. In this case, the dark matter and mediator masses are in the range

$$
m_{\chi} \sim \mathcal{O}(10-100 \mathrm{GeV}) \quad m_{\ell_{i}}+m_{\ell_{j}}<m_{\varphi}<m_{\chi}\left(\frac{2}{3} m_{\chi}\right)
$$

where the value in parenthesis corresponds to the $2 \rightarrow 3$ annihilation.

The $s$-wave annihilation of fermionic dark matter into on-shell spin- 0 mediators was recently studied in refs. $[39,41,84]$. There are three scenarios which give different $s$-wave annihilation modes depending on the parity of $\varphi$ :

1. If $\varphi$ does not have a well defined dark sector parity - that is, both $y_{S}$ and $y_{P} \neq 0$ — then the leading $s$-wave annihilation mode is $\chi \bar{\chi} \rightarrow \varphi \varphi^{*}$. This holds for both real and complex $\varphi$. This is shown in figure $1(\mathrm{a})$.

2. If $\varphi$ is a parity-odd pseudoscalar $\left(y_{S}=0\right)$, then the leading $s$-wave annihilation mode is $\chi \bar{\chi} \rightarrow 3 \varphi$. This is shown in figure $1(\mathrm{~b})$.

3. If $\varphi$ is a parity even scalar $\left(y_{P}=0\right)$, then the leading $s$-wave annihilation mode is $\chi \bar{\chi} \rightarrow 4 \varphi$.

We assume that the mediator mass $m_{\varphi}$ is sufficiently light such that these annihilation modes are accessible. In the present work we focus on the first two of these scenarios since they offer viable candidates for the Fermi $\gamma$-ray excess that are compatible with a thermal relic. If the annihilation results in additional final state mediators, a heavier dark matter mass is required in order to account for the same energy spectra. This implies a smaller galactic halo dark matter number density which in turn forces the annihilation rate to be larger and typically in further tension with a standard thermal relic. While we focus on the case when the present day annihilation rate is compatible with thermal freeze out, we remark that one could relax this requirement — as in Kaplinghat et al. [50] — and assume that the dark sector and Standard Model were reheated to different initial temperatures in the early universe.

Typically one assumes that the mediator decays into Standard Model states: if the mediator is stable, then it is a dark matter component that couples directly to the Standard Model. If, on the other hand, it decays into lighter hidden sector states, then those states are constrained by cosmological limits on the number of light degrees of freedom.

From the point of view of softening the positron spectrum, one may wish to consider mediator masses lighter than one of the leptons to which it couples. In this case, the heavier leptons in figures $1(\mathrm{c}, \mathrm{d})$ are off shell. This forces the $\varphi$ decay to be multi-body and further softens the spectrum. We do not consider this possibility because the constraints from two-body decays of heavy leptons [85, 86], typically imply that the mediator is too long-lived and would be ruled out by cosmological bounds. 


\subsection{Standard model interactions}

Both the dark matter and mediator are taken to be Standard Model singlets. We assume that at low energies, the mediator communicates to the Standard Model only through charged lepton interactions that are chiral and flavor violating,

$$
\mathcal{L}_{\varphi \mathrm{SM}}=g_{i j} \varphi \bar{\ell}_{i} P_{L} \ell_{j}+g_{j i}^{*} \varphi^{*} \bar{\ell}_{j} P_{R} \ell_{i}
$$

where $P_{L, R}=\frac{1}{2}\left(1 \mp \gamma^{5}\right)$ are chiral projection operators. We restrict to the case where $g_{i j}$ is non-zero for only a single pair of distinct flavors, $i \neq j$. For a complex mediator, we choose as a convention, that $\varphi$ rather than $\varphi^{*}$ interacts with a left-handed projection operator. The dominant decay modes of this mediator are shown in figure $1(\mathrm{c}, \mathrm{d})$. We demonstrate below that the chiral flavor violating structure of this interaction softens constraints and better fits the Fermi $\gamma$-ray excess than the Kaplinghat et al. model [52]. This low-energy model is invariant under a global $L_{i}-L_{j}$ symmetry which prohibits most charged leptonflavor violating processes up to corrections from the $W$ interactions and neutrino masses. These are discussed in section 5 .

\subsection{Comments on ultraviolet realizations}

While it is beyond the scope of this phenomenological study, we briefly comment on complete flavor models that may produce this scenario. At the electroweak scale, the interactions in (2.4) can be generated by the gauge-invariant, higher-dimensional operator

$$
\mathcal{L}_{\varphi \mathrm{SM}}^{(\mathrm{EW})}=\frac{c_{i j}}{\Lambda} \varphi H \bar{L}_{i} E_{j}+\text { h.c. },
$$

so that one may identify $g_{i j}=c_{i j} v / \sqrt{2} \Lambda$ and $v$ is the Higgs vacuum expectation value. As an alternative, one could assume (2.4) is generated by $R$-parity violating type superpotential interaction $\lambda_{i j k} L^{i} L^{k} \bar{E}^{k}$ in a supersymmetric extension of the Standard Model, where $i, j, k$ are distinct lepton flavors. This superpotential generates a chiral, flavor-changing coupling mediated by sneutrinos [87, 88].

The electroweak-scale effective operator (2.5) can, in turn, be generated by renormalizable interactions with respect to heavy degrees of freedom. This may be realizable within the Froggatt-Nielsen mechanism, in which a pattern of low-energy flavor-dependent couplings is generated by integrating out heavy degrees of freedom that are charged under the broken flavor symmetries [53]. This mechanism is typically used to explain the pattern of Standard Model Yukawa couplings, but is straightforwardly extended to additionally generate the coupling in (2.5). We reserve the model building aspects for future work, ${ }^{1}$ but remark that one can build such realizations in which (2.5) has only a single dominant entry which is off-diagonal, with all other entries suppressed. In this case, the scale $\Lambda$ of the effective operator can readily be associated with the flavor symmetry breaking scale, and need not be much higher than the electroweak scale, so that the renormalization group corrections to the pattern are very small. While neutrinos may modify the flavor structure of the mediator-Standard Model interactions at two-loop order; we ignore these as they

\footnotetext{
${ }^{1}$ See [80] for discussion of one class of flavor framework.
} 
are typically several orders of magnitude smaller than $g_{i j}$. Supersymmetric flavor models may be a promising direction for such model-building [89-91], especially those in which the flavor symmetry is broken at a higher scale than supersymmetry [92-94]. In this case supersymmetry protects the flavor structure from renormalization group effects down to the supersymmetry breaking scale, and possibly down to the scale of slepton masses.

\subsection{Self-interacting dark matter}

The scalar coupling $y_{S}$ in (2.1) generates a long-range Yukawa potential between dark matter particles. This is the key ingredient for how dark matter self-interactions address small scale structure anomalies [51]. The lightness of the mediator introduces a velocitydependence on the self-scattering cross-section; this affects the dark matter halo profile on the scales of dwarf galaxies, while remaining consistent with constraints from galaxy cluster mergers [95]. In the model by Kaplinghat, Linden, and $\mathrm{Yu}$, a vector mediator, $V$, produces the Fermi $\gamma$-ray excess by the inverse Compton scattering of $\chi \bar{\chi} \rightarrow V V \rightarrow 4 e$ [50]. The vector mass, $m_{V} \sim \mathcal{O}(10-100 \mathrm{MeV})$, and coupling to dark matter, with a transfer cross-section of $\sigma_{T} \sim \mathcal{O}\left(0.5-50 \mathrm{~cm}^{2} / \mathrm{s}\right)$, were found to be of the correct size to realize this self-interacting dark matter target region. This came at the cost of some tension with the thermal relic cross-section, $\langle\sigma v\rangle_{\text {rel. }}$, so that they invoke a different dark sector temperature [96].

In the scalar models here, only the parity-even dark sector interaction in (2.1) mediates a Yukawa potential. The parity-odd interaction mediates a spin dependent potential that scales as $e^{-m_{\varphi} r} / r^{3}$ [97]; this is not expected to have a significant effect on astrophysical dynamics. We thus observe that the scenario with a pure pseudoscalar mediator $\left(y_{S}=0\right)$ does not realize the self-interacting dark matter target region. On the other hand, in the scenario where $\varphi$ has mixed parity, $s$-wave $\chi \bar{\chi} \rightarrow \varphi \varphi^{(*)}$ annihilation depends on both $y_{S}$ and $y_{P}$. This introduces some freedom to choose $y_{S}$ to realize a large self-interaction crosssection and then separately choose $y_{P}$ to select the annihilation cross-section, $\langle\sigma v\rangle$. Our models also differ from Kaplinghat et al. because the minimum mediator mass scale is set by the heavier lepton to which the mediator couples. Thus the lightest mediator mass we consider is $m_{\varphi} \sim m_{\mu}=106 \mathrm{MeV}$ which is accessible for mediators with $\mu e$ couplings. Observe that this mass is near the heavy limit of mediator masses that are compatible with solving small scale structure anomalies [51]. We then expect that the case where the mediator couples to a $\tau$ are typically incompatible with the self-interacting dark matter target region.

While a detailed study of the dark matter self-interactions in this model is beyond the scope of this paper, the benchmark results in ref. [51] already demonstrate the key properties. We specifically note that the dark matter and mediator masses considered here populate the numerically difficult resonant regime where consistency with the selfinteracting dark matter target region is plausible but very sensitive to the precise values of

$m_{\chi, \varphi}$. For this reason, in this manuscript we focus on the compatibility of the Fermi $\gamma$-ray excess in our scenario with the thermal relic cross-section without invoking a different dark sector temperature. We leave the details of the dark sector self-interactions - which we emphasize are automatic in our constructions - for separate work. 


\section{The Fermi-LAT $\gamma$-ray excess}

\subsection{Photons from leptons}

The spectrum of photons to be identified with the Fermi $\gamma$-ray excess originate from two sources:

1. Prompt photons from the final state leptons or

2. Up-scattered starlight from the inverse Compton scattering (ICS) of $e^{ \pm}$produced through the $\varphi$ decay.

This is in contrast to models where dark matter annihilates predominantly into quarks or gluons. In that case the photons are a result of $\pi^{0} \rightarrow \gamma \gamma$ decays from the showering and hadronization of the final state partons. Since the $\tau$ has a large hadronic decay width $(\sim 65 \%)$, its spectrum of prompt photons is similar to that of quarks and gluons. In contrast, electrons and muons (and leptonically decaying taus) typically produce a smaller flux of prompt photons, but can yield a large number of up-scattered photons from inverse Compton scattering in a stellar radiation field. The scattered photon energy, $E_{\gamma}^{\prime}$ can be approximated in terms of the incoming photon energy, $E_{\gamma}$ and the scattering electron energy, $E_{e}$

$$
E_{\gamma}^{\prime} \approx\left(\frac{E_{e}}{m_{e}}\right)^{2} E_{\gamma}
$$

In the $\chi \bar{\chi} \rightarrow V V \rightarrow 4 e$ model of Kaplinghat et al., $m_{\chi} \sim 10 \mathrm{GeV}$ so that $\left(E_{e} / m_{e}\right) \sim 10^{4}$ and $\mathcal{O}(10 \mathrm{eV})$ starlight is then up-scattered to $\mathcal{O}(\mathrm{GeV})$, corresponding to the characteristic scale of the Fermi-LAT $\gamma$-ray excess. In contrast, the $e^{ \pm}$energy spectrum resulting from the scalar mediator decays $\varphi \rightarrow \bar{\ell}_{i} \ell_{j}(i \neq j)$ and subsequent decays to electrons is softer as part of the energy is deposited in neutrinos. The average $e^{ \pm}$energies in the $e \mu, e \tau, \mu \tau$ scenarios in the limit $m_{\ell} \ll m_{\varphi}$ are suppressed by $\sim \frac{2}{3}, \frac{11}{18}, \frac{5}{18}$ with respect to the $\varphi \rightarrow e^{+} e^{-}$scenario, where we only account for leptonic $\tau$ decays. We therefore expect that the average ICS photon energy to be approximately an order of magnitude softer in the flavor-violating case.

In order to properly estimate the photon spectrum, we use the Mathematica package PPPC $[98,99]$. As an input, PPPC requires the flavor dependent energy spectrum distributions of the leptonic annihilation products,

$$
\frac{d N_{\ell_{j}}}{d E_{\ell_{j}}}=\int d E_{\varphi} \frac{d N_{\varphi}}{d E_{\varphi}} \frac{d N_{\ell_{j}}^{\varphi}\left(E_{\varphi}\right)}{d E_{\ell_{j}}}
$$

where $d N_{\varphi} / d E_{\varphi}$ is the spectrum of mediators and $d N_{\ell_{j}}^{\varphi}\left(E_{\varphi}\right) / d E_{\ell_{j}}$ is the spectrum of $j$-type leptons produced in the decay of a mediator with energy $E_{\varphi}$. For annihilation into two mediators, the $\varphi$ energy spectrum is monochromatic, $d N_{\varphi} / d E_{\varphi}=\delta\left(E_{\varphi}-m_{\chi}\right)$, so that the boost of lepton energies from the $\varphi$ rest-frame is

$$
E_{\ell}=\gamma E_{\ell}^{0}+\sqrt{\gamma^{2}-1}\left|\mathbf{p}_{\ell}^{0}\right| \cos \theta
$$


where $E_{\ell}^{0}$ and $\mathbf{p}_{\ell}^{0}$ are the lepton energy and three-momentum in the $\varphi$ frame, and $\gamma=$ $E_{\varphi} / m_{\varphi}$ is the boost to the lab frame. The lepton energy distribution is then box-shaped because the cosine of the azimuthal angle $\cos \theta$ is uniformly distributed over its range. For annihilation into three mediators, these distributions are implemented following the discussion in ref. [41]; see [42, 100] for related discussions on cascade decays from mediators.

The secondary photon spectrum is,

$$
\frac{d N_{\gamma}}{d E_{\gamma}}=\sum_{j} \int d E_{\ell_{j}} \frac{d N_{\ell_{j}}}{d E_{\ell_{j}}} \frac{d N_{\gamma}^{\ell}\left(E_{\ell_{j}}\right)}{d E_{\gamma}},
$$

where $d N_{\gamma}^{\ell}\left(E_{\ell}\right) / d E_{\gamma}$ is the spectrum of photons produced from a lepton $\ell$ with energy $E_{\ell}$. These are extracted from Pythia [101] and encoded in PPPC.

PPPC decays muons and taus and calculates the total $e^{ \pm}$energy spectrum at the galactic center region. In turn, this spectrum is used as an initial condition in the calculation of the differential $e^{ \pm}$flux $\frac{d \phi_{e}^{ \pm}}{d E_{e}}\left(E_{e}, \vec{x}\right)$ which determines the inverse Compton scattering spectrum of $\gamma$-rays [49]. In calculating the ICS spectrum of photons, we use the MED set of diffusion parameters as described in [102] and a Navarro-Frenk-White (NFW) halo profile for the dark matter distribution with inner profile slope of $\gamma_{\mathrm{NFW}}=1.0$ and a local dark matter density of $\rho_{\odot}=0.4 \mathrm{GeV} \mathrm{cm}^{-3}$ [103]. If one uses a contracted NFW profile, as suggested in refs. $[14,15,17]$ and used by Kaplinghat et al., the required cross-section is reduced by a factor of $\sim 3$ to match the $\gamma$-ray excess intensity. Varying the diffusion parameters across the range of uncertainties as described in [102] does not significantly affect the resultant ICS spectrum. However, their theoretical uncertainties are set by modeling the propagation from across much longer ranges than our region of interest. The actual range of uncertainties on diffusion parameters for galactic center are unknown and may well be much larger.

\section{$3.2 \quad$ Fit to Fermi $\gamma$-ray excess}

Figures 2 and 3 show the photon spectrum prediction for the $15^{\circ} \times 15^{\circ}$ region of the sky centered at galactic coordinates $(l, b)=(0,0)$. We considered each lepton flavor model and each dark matter annihilation mode separately. We tested several $m_{\chi}$ benchmarks, each of which is plotted in a different color. In each benchmark, the range of $m_{\varphi}$ masses considered is accounted for by the thickness of each plotted color. The color edges interpolate the range $m_{\varphi} \in\left[m_{\ell}^{\text {heavy }}, m_{\chi}\left(\frac{2}{3} m_{\chi}\right)\right]$ for the two-(three-) $\varphi$ annihilation mode. Heavier mediators typically bend the spectrum to be slightly harder. Each plot is shown with a fixed benchmark annihilation cross-section. The spectrum scales linearly with this cross-section $\langle\sigma v\rangle$ and quadratically with the local dark matter density $\rho_{\odot}$. To aid in rescaling estimates, we also provide the cross-sections for a contracted NFW profile $\gamma_{\mathrm{NFW}}=1.2$ which produce the same curves. Note that the contracted profile only contains a rescaling by the $J$-factor. Because the interstellar radiation field is not uniform in the region of interest, it is possible that the contracted profile may lead to a change in the ICS spectrum. The range of cross-sections can be interpreted as an estimate of uncertainty when comparing to the thermal relic cross-section. 

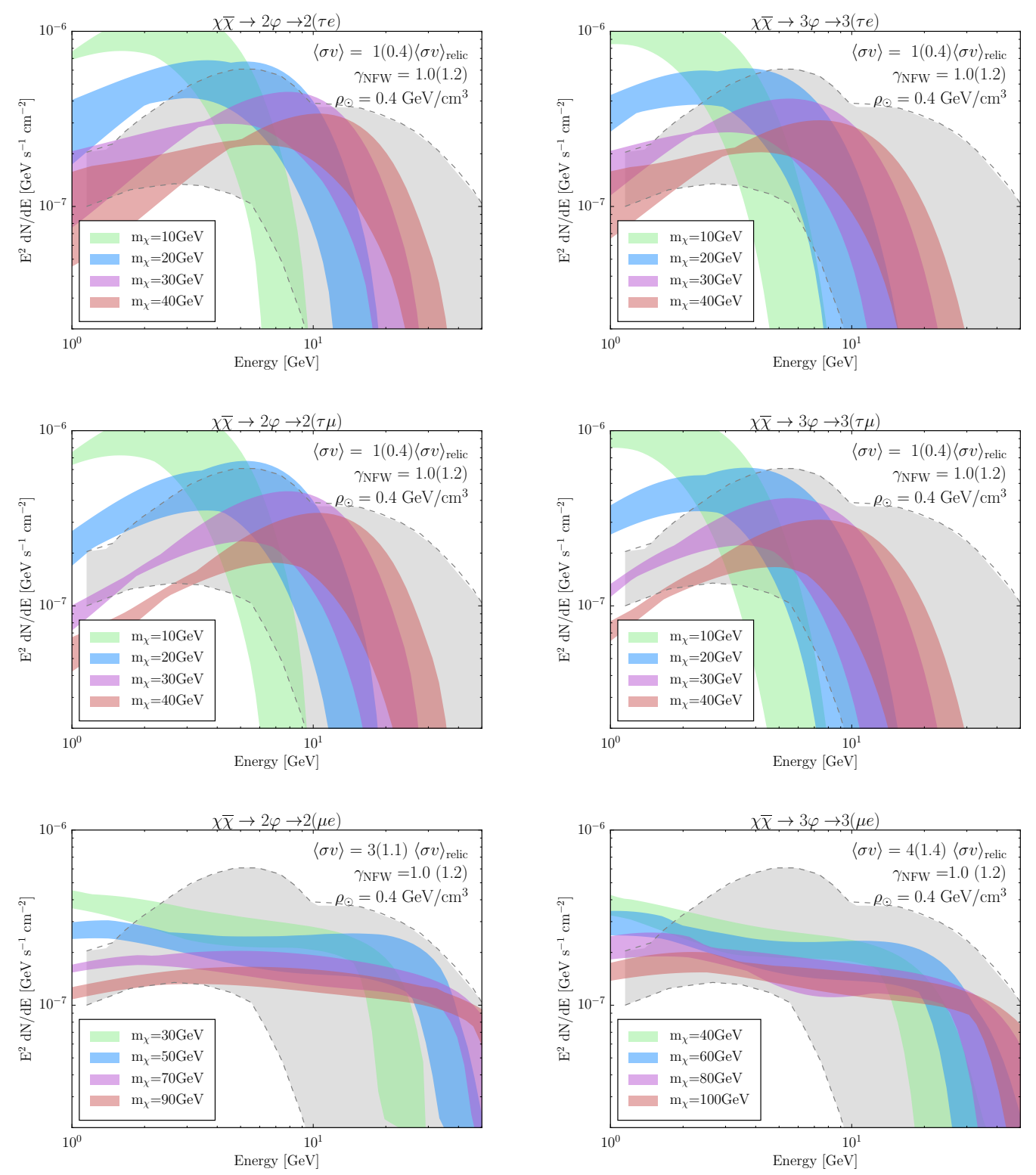

Figure 2. The predicted combined prompt and ICS gamma-ray spectra for each scenario: $\chi \bar{\chi} \rightarrow 2 \varphi$ (left) and $\chi \bar{\chi} \rightarrow 3 \varphi$ followed by the lepton-flavor violating decays indicated in each panel. The grey shaded region represents the Fermi collaboration's $\gamma$-ray excess spectrum bounded by its estimated systematic error when fit as a parameterized form to the entire energy range of the data. Each colorcoded band corresponds to a set of $\left\{m_{\chi}, m_{\varphi}\right\}$ with $m_{\varphi}$ varying in the range $\left[m_{\ell_{\text {heavy }}}, m_{\chi}\right]$ (left) and $\left[m_{\ell_{\text {heavy }}}, \frac{2}{3} m_{\chi}\right]$ (right). The spectra are calculated assuming a halo profile slope of $\gamma_{\mathrm{NFW}}=1.0$ and the annihilation cross sections indicated in each figure. For a steeper halo profile of $\gamma_{\mathrm{NFW}}=1.2$, cross sections are a factor of $\sim 3$ smaller. 

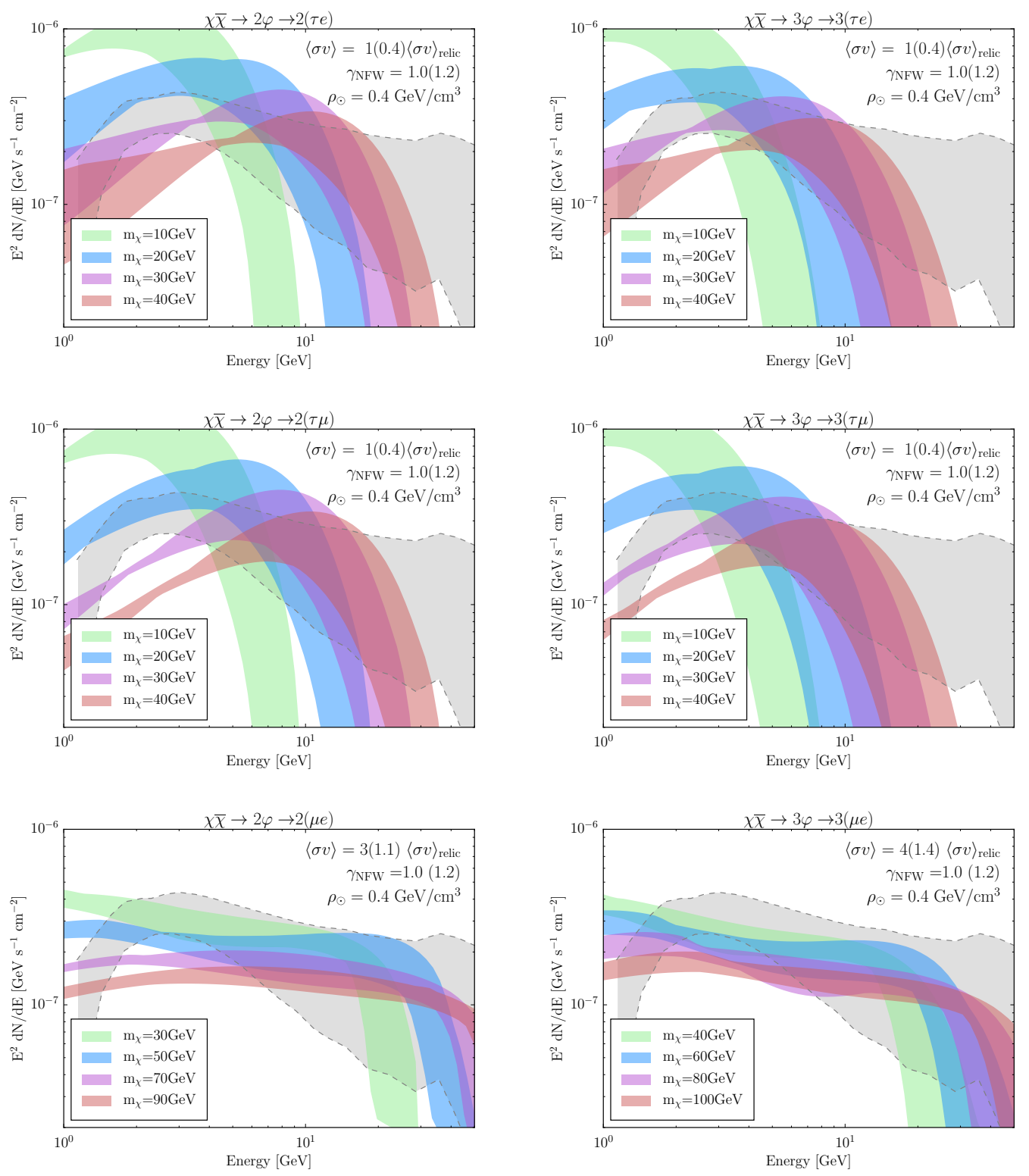

Figure 3. Same as figure 2, but now the grey shaded region represents the $\gamma$-ray excess spectrum bounded by its estimated systematic error when fit in independent energy bins, as reported by the Fermi collaboration. Our predicted combined prompt and ICS gamma-ray spectra for each scenario: $2 \rightarrow 2$ (left) and $2 \rightarrow 3$ (right) $\chi \chi$ annihilations to $\varphi$ s, followed by one of the following decays: $\varphi \rightarrow \tau e$ (up), $\tau \mu$ (middle), and $\mu e$ (bottom). Each color-coded band corresponds to a set of $\left\{m_{\chi}, m_{\varphi}\right\}$ with $m_{\phi}$ varying in the range $\left[m_{\ell_{\text {heavy }}}, m_{\chi}\right]$ (left) and $\left[m_{\ell_{\text {heavy }}}, \frac{2}{3} m_{\chi}\right]$ (right). 
For comparison to the observed Fermi-LAT spectrum, we plot in grey the systematic error band of the $\gamma$-ray excess spectrum as defined in the Fermi collaboration study of the $\gamma$-ray emission from the galactic center [20]. The Fermi collaboration provides two different estimates of the excess $\gamma$-ray spectrum and its systematic uncertainty:

1. The first fits the excess as a parameterized exponential cutoff spectrum across the entire energy range of the data. This is shown in figure 2.

2. The second fits the $\gamma$-ray spectrum in independent energy bins. This is shown in figure 3 .

Flavor-dependence of $\gamma$-ray spectra. The $\mu e$ final states result in much harder $\gamma$ ray spectra than $\tau e$ or $\tau \mu$ final states. In the case of $\mu e$ final states, the ICS contributes the majority of the $\gamma$-ray flux at lower, $\mathcal{O}(1 \mathrm{GeV})$, energies while the prompt contribution dominates at higher, $\mathcal{O}(10 \mathrm{GeV})$, energies. In contrast, the ICS $\gamma$-ray flux in the cases of $\tau e$ and $\tau \mu$ final states only constitutes a small fraction of the low energy spectrum, while the total signal is dominated by the prompt photon 'bump', which peaks between $2-10 \mathrm{GeV}$ before a spectral cutoff. This is because the hadronic $\tau$-decays allow for these annihilation channels to produce a much higher flux of prompt photons.

Comparison to Fermi spectra. Our models are able to reasonably reproduce the parameterized Fermi $\gamma$-ray excess spectrum in figure 2. The $\tau e$ and $\tau \mu$ model achieve this with dark matter masses of $m_{\chi} \sim 20-40 \mathrm{GeV}$, while for the $\mu e$ case, slightly higher masses of $m_{\chi} \sim 40-100 \mathrm{GeV}$ are required. The $\gamma$-ray excess is primarily produced through prompt emission from $\tau$ decays in the $\tau \mu$ and $\tau e$ models, whereas it is primarily produced through ICS in the $\mu e$ model. The dark matter mass for $\mu e$ final states must therefore be higher than the $\tau e / \tau \mu$ cases in order for the resulting electron spectrum to be hard enough to produce the Fermi $\gamma$-ray spectrum through ICS. We note that although the $\gamma$-ray spectra produced in the $\mu e$ models may lie within the systematic error band defined by the Fermi collaboration's parametric fits, they are generally harder at high energies and do not have the characteristic peak at $\sim 2-4 \mathrm{GeV}$ that is typically found in template analyses of the $\gamma$-ray excess. The 'cinched' shapes of the enveloped range of $\gamma$-ray emission in the $\tau e$ channels of figures 2-3 arise due to a sampling effect: the spectra of the outgoing $\tau$ 's and $e$ 's do not change significantly until the mediator mass approaches its allowed minimum (the heavier lepton mass). As the enveloped region is defined using four linearly spaced values of $m_{\phi}$, the spectra for the three heavier mediator masses in the $\tau e$ case are very similar to each other.

On the other hand, figure 3 shows that our computed spectra are worse at fitting the Fermi collaboration's $\gamma$-ray spectra obtained through bin-by-bin fits. The spectrum derived through fitting the data in individual energy bins displays an extended, power-law-like tail at energies $\geq 10 \mathrm{GeV}$; this was observed in $[18,19]$ and has recently been explored further in [104]. Our theoretical $\gamma$-ray spectra all cut off sharply around $\mathcal{O}\left(m_{\chi}\right)$ and thus cannot reproduce this spectral feature. One can interpret the difference between the parameterized and bin-by-bin fits as a qualitative assessment of the uncertainty in the target region for the spectral fit. 
Compatibility with relic abundance. For $\tau e$ and $\tau \mu$ final states, we are able to produce the observed Galactic Center excess flux with an annihilation cross-section $\langle\sigma v\rangle$ roughly equal to the relic density cross-section, (2.2). For $\mu e$ final states, the annihilation cross-section must be $3-4$ times higher than the canonical relic cross-section in order to match the intensity of the Fermi Galactic Center excess. This is again related to the fact that there are substantially fewer prompt photons in the $\mu e$ scenario, hence the larger annihilation rate needed to account for the excess. We point out, however, the $\mu e$ states are brought back into consistency with (2.2) if one instead invokes a contracted NFW profile.

Dwarf spheroidal bounds. Dwarf spheroidals are satellite galaxies that are rich in dark matter but have relatively little stellar matter. As a result, they typically set the strongest bounds on models of the galactic center excess that rely on prompt photon emission [45]. This is avoided when the $\sim \mathrm{GeV}$ photons are produced though the inverse scattering of starlight because the dwarfs have a weak interstellar radiation field. Thus the $\mu e$ models are able to completely evade the dwarf bounds.

For $\tau e$ and $\tau \mu$ channels, the decay into two differently flavored leptons means that the cross-section for annihilation to $\tau$ 's is half of the total annihilation cross-section. We also note that the range of dark matter particle masses considered here for annihilations to mediators are generally higher than the best-fit masses in the case of direct annihilation. This is because either four or six SM leptons are produced per annihilation in these models instead of two in the direct scenario. The dwarf constraints on the annihilation crosssection into $\tau$ 's are roughly $\sim 1.5-2$ times weaker at $m_{\chi} \sim 20-40 \mathrm{GeV}$ compared to $m_{\chi} \sim 10 \mathrm{GeV}$, which is often quoted as the best-fit mass for direct annihilations into $\tau$ 's. These two effects combine to partially alleviate the existing tensions with dwarf constraints on prompt $\gamma$-ray flux from annihilation into $\tau$ 's; the cross-sections required in our model for $\tau e$ and $\tau \mu$ channels are within $1 \sigma$ of the limits from ref. [45].

Comparison to Kaplinghat et al. We briefly compare our results to the $\chi \bar{\chi} \rightarrow V V \rightarrow$ $4 e$ scenario; in doing so, we may highlight the differences in the lepton-flavor violating case and the role of uncertainties in astrophysical parameters. Kaplinghat et al. [50] found that the annihilation mode to $4 e$ fits the Galactic Center excess for an annihilation crosssection of $\langle\sigma v\rangle \approx\langle\sigma v\rangle_{\text {rel. }} / 7$. By comparison, our 2( $\left.\mu e\right)$ annihilation mode is found to fit with $\langle\sigma v\rangle \approx 3\langle\sigma v\rangle_{\text {rel. }}$. The difference between these factors are predominantly from three sources:

1. Our result uses a standard $\gamma_{\mathrm{NFW}}=1.0$ dark matter halo profile while Kaplinghat et al. use a contracted $\gamma_{\mathrm{NFW}}=1.2$ profile. As shown in the plot, the difference in $J$ factors is approximately a factor of 3 .

2. The discussion in section 3.1 shows that electrons and muons produce very different ICS spectra. This is in contrast to prompt photon production where the mass difference can be ignored at sufficiently high energies. This introduces an $\mathcal{O}$ (few) difference between the flavor violating and flavor conserving modes.

3. Finally, there are some $\mathcal{O}$ (few) differences in the target region, Kaplinghat et al. use the fit by [17] while we use the envelopes from the Fermi collaboration [20]. 


\section{The AMS-02 $e^{ \pm}$spectrum}

The AMS-02 experiment may be capable of detecting electrons and positrons produced in dark matter annihilations [105]. AMS observations thus far have found that the $e^{ \pm}$spectra are smoothly varying, with no line-like or bump features [106]. We constrain the range of particle properties allowed in our model by requiring that the flux of $e^{ \pm}$produced through annihilations and propagated to Earth's position in the Milky Way must be low enough to avoid producing any such features in the observed $e^{ \pm}$spectra. To do so, we use the DRAGON 3D cosmic ray propagation code along with the propagation setup described in ref. [107]. The diffusion coefficient is assumed to depend on particle rigidity as

$$
D(\rho)=\beta^{-0.4} D_{0}\left(\frac{\rho}{\rho_{0}}\right)^{\delta} \quad \text { with } D_{0}=3 \times 10^{28} \mathrm{~cm}^{2} \mathrm{~s}^{-1}, \rho_{0}=3 \mathrm{GV}, \delta=0.6 .
$$

We assume the same halo parameters and annihilation cross-section for each case of lepton final states and number of mediators as in the previous section.

Figure 4 shows the expected $e^{ \pm}$energy spectrum from $\chi \bar{\chi}$ annihilation to $\varphi$ followed by the decay $\varphi \rightarrow \bar{\ell}_{i} \ell_{j}$. Following the notation of the previous section, results are presented for each annihilation mode and each of the three $\varphi$ decay models separately. We fix the dark matter annihilation rate of each model to roughly match the observed Galactic Center $\gamma$-ray excess flux. We regard a model as consistent with the AMS observations if for all energies, the $e^{ \pm}$flux predicted by the model is lower than the total size of the error bars given in ref. [106] for the $e^{+}$and $e^{-}$binned fluxes at that energy.

We find that for $\tau e$ and $\tau \mu$ final states, dark matter masses above $\sim 20 \mathrm{GeV}$ are not excluded by AMS observations, and can also produce a $\gamma$-ray signal consistent with the Fermi excess. For $\mu$ final states, all potential $\mathrm{e}^{ \pm}$spectra studied here are in tension with the AMS positron bounds when $\gamma_{\mathrm{NFW}}=1.0$. This tension may be reduced by either

1. increasing the dark matter mass beyond $90-100 \mathrm{GeV}$, or

2. considering of a steeper dark matter halo density profile.

Increasing the mass comes at the expense of hardening the $\gamma$-ray signal and introduces tension with the Fermi result. On the other hand, a slightly steeper NFW inner profile slope or $\gamma_{\mathrm{NFW}}=1.1-1.3$ was suggested in $[15,17]$ for the Fermi $\gamma$-ray excess. When using a steeper profile, the annihilation cross-section needed to reproduce the observed excess brightness decreases by up to a factor of $\sim 5$. Since the local dark matter density is held fixed as the density profile slope changes, this lower annihilation rate results in a decrease in the dark matter contribution to the AMS $e^{ \pm}$spectrum. The potential dark matter contribution to the local $e^{ \pm}$spectrum is dominated by the flux from annihilations near the solar neighborhood; changes to the profile near the galactic center have little effect on this measurement. The dashed lines in the $\mu e$ plots of figure 4 show the upper contour of the $e^{ \pm}$spectra for the estimated reduced annihilation cross-sections with a contracted $\gamma_{\mathrm{NFW}}$ profile. One can see that the factor of $\sim 3$ in the cross-section allows these modes to avoid the AMS $e^{+}$bound. 

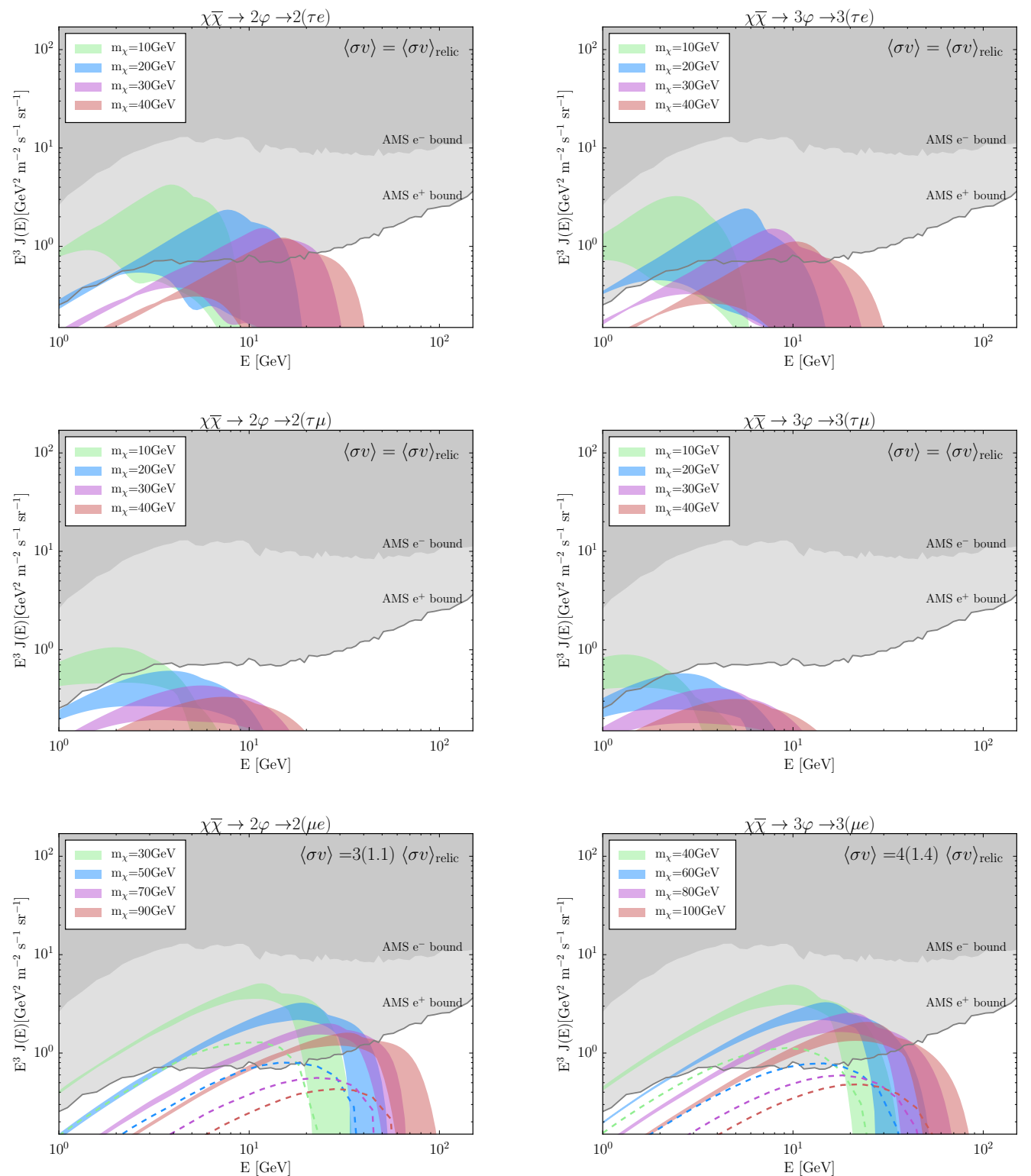

Figure 4. Predicted $e^{ \pm}$spectra at Earth's position for each scenario: $\chi \bar{\chi} \rightarrow 2 \varphi$ (left) and $\chi \bar{\chi} \rightarrow 3 \varphi$ (right) followed by one the lepton-flavor violating decays indicated in each panel. The grey shaded region represents the bounds from AMS-02 electron and positron spectra while each color-coded band corresponds to a set of $\left\{m_{\chi}, m_{\phi}\right\}$ with $m_{\phi}$ varying in the range $\left[m_{\ell_{\text {heavy }}}, m_{\chi}\right]$ (left) and $\left[m_{\ell_{\text {heavy }}}, \frac{2}{3} m_{\chi}\right]$ (right). The dashed lines shown in the bottom row correspond to the prediction for AMS-02 spectra if an NFW profile slope of $\gamma_{\mathrm{NFW}}=1.2$ is assumed. This leads to a lower annihilation cross section of 1.1 (1.4) times the relic density for $\chi \bar{\chi} \rightarrow 2(3) \varphi$. 


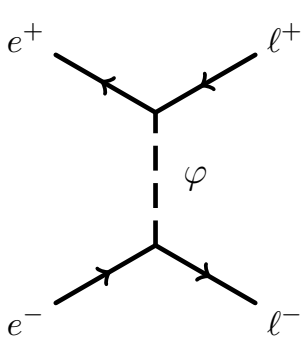

(a) $A_{\mathrm{FB}}^{\ell}$

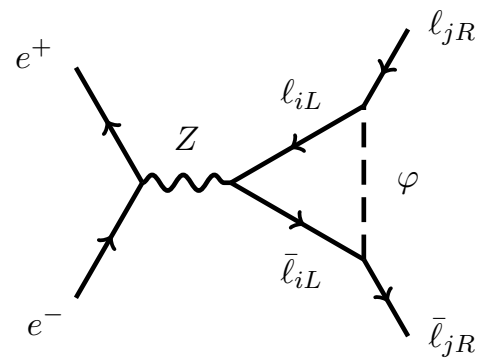

(b) $Z$ couplings

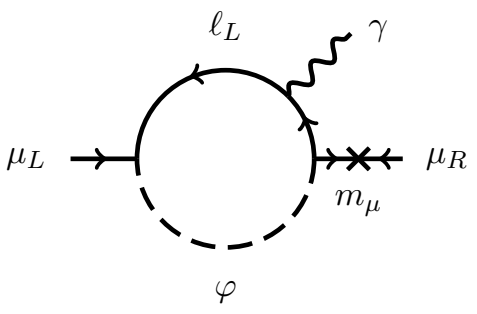

(c) $(g-2)_{\mu}$

Figure 5. Diagrams demonstrating possible constraints on the Standard Model couplings of the $\varphi,(2.4)$. In (c), arrows represent helicity to show that an external mass insertion is required.

\section{Constraints on standard model couplings}

The discussions in sections 3-4 focused on the target region and constraints on the dark sector couplings, (2.1), with the assumption that the mediator decays are sufficiently prompt on astrophysical scales. In this section we review the constraints on the Standard Model couplings, (2.4), that control that decay length. We emphasize that by virtue of the hidden sector scenario, these couplings can be taken to be very small to avoid the bounds here without causing the decay length to distort the Fermi or AMS analyses above.

\subsection{Photon lines}

Mediators may decay into photon pairs, $\varphi \rightarrow \gamma \gamma$, if flavor-conserving couplings are generated. These decays would be seen in the galactic $\gamma$-ray spectrum [108]. Such couplings are assumed to be negligible as they are only generated by $\left(L_{i}-L_{j}\right)$-breaking effects.

\subsection{Electroweak precision measurements}

Electron colliders are able to probe the chiral structure of new physics through the forwardbackward asymmetries of $e^{+} e^{-} \rightarrow f \bar{f}$ scattering [109],

$$
A_{\mathrm{FB}}^{f}=\frac{\sigma_{>}\left(e^{+} e^{-} \rightarrow f \bar{f}\right)-\sigma_{<}\left(e^{+} e^{-} \rightarrow f \bar{f}\right)}{\sigma_{>}\left(e^{+} e^{-} \rightarrow f \bar{f}\right)+\sigma_{<}\left(e^{+} e^{-} \rightarrow f \bar{f}\right)},
$$

where $\sigma_{>(<)}$refers to the forward (backward) cross-section where the azimuthal angle of the $f$ with respect to the $e^{-}$has positive (negative) cosine. The $t$-channel exchange of a mediator modifies the forward-backward asymmetry relative to its Standard Model value; this is shown in figure 5a. Observations of $A_{\mathrm{FB}}^{\ell}$ for $\ell=\mu, \tau$ therefore constrain the couplings and mass of $\varphi$. It is straightforward to translate such bounds on supersymmetric $R$-parity violating models to our scenario, by identifying the sneutrino with $\varphi$, and decoupling the rest of the supersymmetric spectrum. Comparing with [110, 111], we find

$$
g_{\mu e, e \mu}<2.5 \times 10^{-3}\left(\frac{m_{\varphi}}{\mathrm{GeV}}\right) \quad g_{\tau e, e \tau}<1.1 \times 10^{-3}\left(\frac{m_{\varphi}}{\mathrm{GeV}}\right) .
$$

The chiral couplings of the $Z$ boson are also precisely measured by SLD and LEP through $e^{+} e^{-} \rightarrow Z \rightarrow \ell^{+} \ell^{-}$[112]. In our model, vertex corrections with an internal $\varphi$ 
line will mix the left-handed $\ell_{j}$ and right-handed $\ell_{i}$ couplings; this is shown in figure 5b. Altmannshofer et al. recently performed full analysis of these couplings, including error correlations in the couplings, for the case a $\tau \mu$ lepton-flavor violating spin-1 boson [55]. They found that the bound from this measurement is typically much weaker than the anomalous magnetic moment of the muon. We thus assume that these constraints are subdominant to (5.2) for the $\mu e$ and $\tau e$ couplings and the muon magnetic moment (discussed below) for the $\tau \mu$ couplings of $\varphi$.

Other subdominant constraints include corrections to the $Z$ and $W$ widths from the on-shell emission of $\varphi$ off a charged lepton decay product [113], corrections to the PeskinTakeuchi parameters (which begin at two-loop order) [114], and contribution to the highly suppressed multi-lepton decay modes of charged kaons.

\subsection{Lepton anomalous dipole moments}

The anomalous electric- and magnetic-dipole moments of leptons, place strict constraints on light new physics [115-119], including lepton flavor violating new physics [120]. The interaction structure of (2.4), assures that with a single chiral coupling, a complex phase in $g_{i j}$ can be rotated away in the $\left(L_{i}-L_{j}\right)$-symmetric limit. As a result, electric dipole moments do not play a role in constraining the allowed parameter-space of the coupling. On the other hand, contributions to magnetic dipole operators are generated already at the one-loop level, and are experimentally constrained. These operators involve both left- and right-lepton chirality states, and therefore require mass insertions for (2.4) to contribute. The contribution of (2.4) to the anomalous magnetic dipole moment, $a_{\ell_{j}}=\frac{1}{2}(g-2)_{\ell_{j}}$, is

$$
\Delta a_{\ell_{j}}=\frac{m_{\ell_{j}}}{16 \pi^{2}} \sum_{i=1}^{3} \int_{0}^{1} d x(1-x)^{2} \frac{x m_{\ell_{j}} S_{i}+m_{\ell_{i}} P_{i}}{x m_{\varphi}^{2}+(1-x) m_{\ell_{i}}^{2}-x(1-x) m_{\ell_{j}}^{2}},
$$

where $\Delta a_{\ell}=a_{\ell}^{\exp }-a_{\ell}^{\mathrm{SM}}$ is the deviation from the Standard Model prediction, $S_{i}=$ $\left|g_{i j}\right|^{2}+\left|g_{j i}\right|^{2}$, and $P_{i}=g_{i j}^{*} g_{j i}+g_{j i}^{*} g_{i j}$; see also [121] where $\varphi$ is identified with a sneutrino with an $R$-parity-violating interaction.

The bounds on the electron $[122,123]$ and muon [124] magnetic moments are

$$
\Delta a_{e}=-1.05(0.82) \times 10^{-12} \quad \Delta a_{\mu}=288(80) \times 10^{-11},
$$

Note that the central value of $\Delta a_{e}$ is negative, and cannot be accounted for by (5.3) under the single coupling $g_{i j}$ assumption. The chirality flip required to generate the dipole operator can only occur on an external line, as in figure $5 \mathrm{c}$. The bounds from magnetic dipole moments, and the forward-backward asymmetryare plotted in figure 6. Following [125], we plot the exclusion bounds for $\Delta a_{e}$ for various confidence levels, in the parameter-space region where it is positive. We also plot the preferred parameter-space region to account for the $2.6 \sigma$ anomaly in $(g-2)_{\mu}$ [124].

\subsection{Charged lepton flavor violation}

The $L_{i}-L_{j}$ symmetry suppresses traditional signatures of charged lepton flavor violation [126]. While the contributions to most tree-level processes are zero, symmetry breaking 

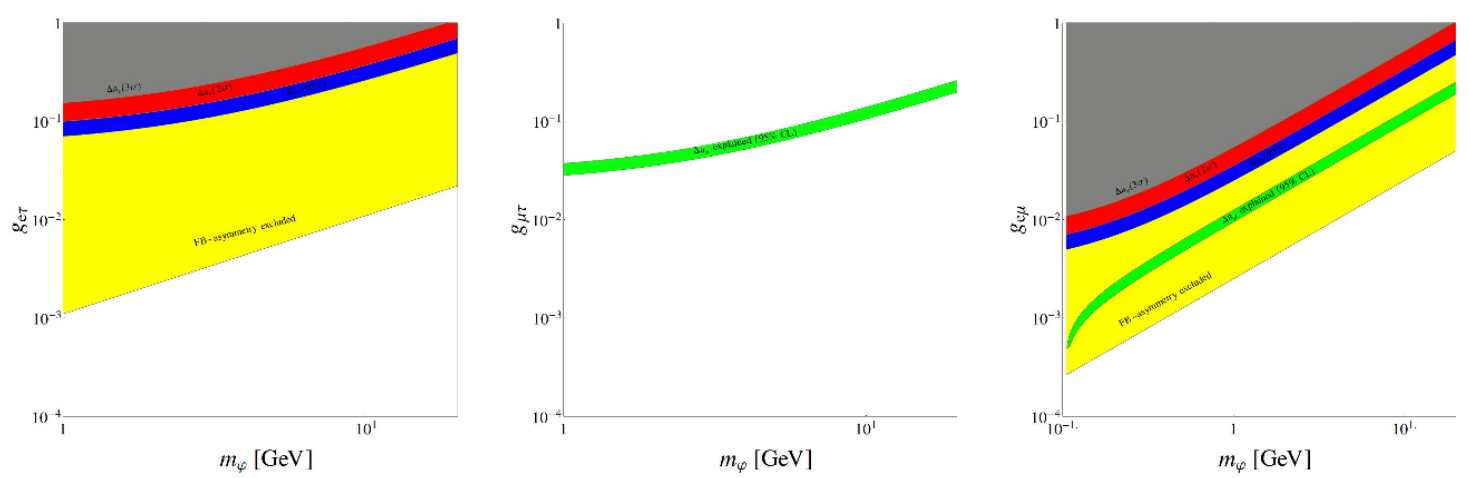

Figure 6. Excluded and preferred parameter-space of the flavor-conserving observables: $\Delta a_{e}, \Delta a_{\mu}$, and $A_{\mathrm{FB}}^{f}$ in the $g_{i j}-m_{\varphi}$ plane. The entire $\Delta a_{e, \mu}$ excluded and preferred region falls into the domain excluded by the forward-backward asymmetry (yellow). The $\mu \tau$ model has no constraint from the forward-backward asymmetry and can account for both the $(g-2)_{\mu}$ excess and the Fermi $\gamma$-ray excess.

effects can still enter through loop-induced interactions of charged leptons with neutrinos and $W$-bosons. These effects are, however, highly suppressed due to the loop-nature, the $W$-mass, and the leptonic GIM-mechanism involving the small neutrino masses.

- Lepton radiative decays, $\ell_{i} \rightarrow \ell_{j} \gamma[127,128]$. Thhe one-loop contribution to these flavor-changing dipole operators vanishes. The leading $\varphi$ contribution starts at threeloop order and is highly suppressed.

- $\mu \rightarrow e$ conversions in heavy nucleus. [129-131] Since $\varphi$ does not directly couple to nuclei, the leading contributions appear from the flavor-changing dipole operator so that $\operatorname{Br}(\mu \mathrm{Nuc} \rightarrow \mathrm{e} \mathrm{Nuc}) \approx \alpha_{E M} \times \operatorname{Br}(\mu \rightarrow e \gamma)$. This dipole, however, vanishes due to the chiral nature of the $\varphi$ interactions.

- Multibody rare lepton decays, $\ell_{i} \rightarrow \ell_{j} \ell_{k} \bar{\ell}_{k}[132,133]$ and $\ell_{i} \rightarrow \ell_{j} \ell_{k} \bar{\ell}_{k} \nu \bar{\nu}$ [134]. As $\varphi$ has no tree-level flavor-conserving interactions the first process cannot proceed via tree-level interactions. The coupling constraints of the preceding section then imply that any induced flavor-conserving coupling is negligible. If one allows for neutrino final states as in the second process, tree-level $W$-interactions then violate $L_{i}-L_{j}$ and $\varphi$ can participate in mediating the second type processes. In this case, both $\varphi$ and an intermediate lepton state must go off-shell making these types of contributions highly suppressed. We have numerically verified that such contributions are negligible, even for $g_{i j}=1$, and for $m_{\varphi} \gtrsim m_{\ell_{j}}(j>i)$.

- Muonium oscillation, $M \leftrightarrow \bar{M}, M=\bar{\mu} e$ [135]. In the absence of flavor-conserving $\varphi$ couplings, muonium oscillations place strong bounds on $\mu$ flavor violation. In our case, the $L_{i}-L_{j}$ symmetry forbids tree-level contributions and $\varphi$ interactions can only decorate the already highly suppressed one-loop SM contributions. ${ }^{2}$

\footnotetext{
${ }^{2}$ On the other hand, a real scalar cannot carry spurious $L_{\mu}-L_{e}$ charge, in which case the bound is rather strong [136] $\left.g_{\mu e, e \mu}\right|_{\varphi \in \mathbb{R}}<4.4 \times 10^{-4}\left(\frac{m_{\varphi}}{\mathrm{GeV}}\right)$.
} 


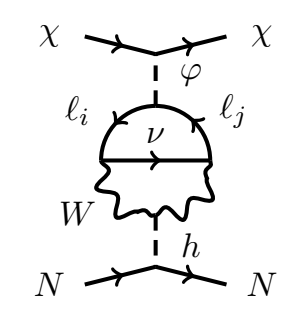

(a) direct detection

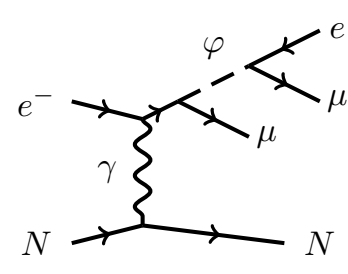

(b) $\varphi$ bremsstrahlung

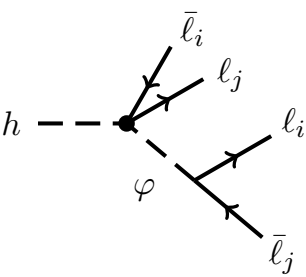

(c) $h \rightarrow \ell_{i}^{+} \ell_{i}^{-} \ell_{j}^{+} \ell_{j}^{-}$

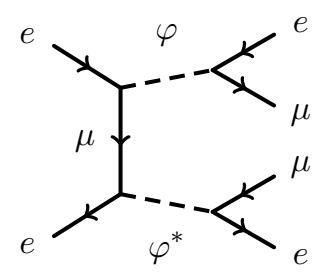

(d) $e^{+} e^{-} \rightarrow \ell_{i}^{+} \ell_{i}^{-} \ell_{j}^{+} \ell_{j}^{-}$

Figure 7. Diagrams demonstrating possible search strategies at direct detection experiments, fixed target experiments, hadron colliders, and lepton colliders.

\subsection{Direct detection}

In the case of a flavor-conserving leptophilic mediator coupling to $\mathcal{O}(100 \mathrm{GeV})$ dark matter, the interaction with the direct detection target nuclei is loop suppressed [137]. In our lepton flavor-violating scenarios, these interactions are further suppressed due to the flavor violating nature of the interaction. Because $\varphi$ is neutral and only couples to differentflavor leptons and the only source for lepton flavor violation in the Standard Model is the $W$ boson, the mixing between the $\varphi$ and, say, a Higgs boson only occurs at two-loop order. This is shown in figure 7a. Note that this diagram is further suppressed by mass insertions of an internal charged lepton and a neutrino due to the left-handed $W$ coupling relative to the chiral $\varphi$ coupling and the GIM mechanism.

\subsection{Dark photon searches}

The lower mass range of $\varphi$ is comparable to the range considered by dark photon searches. We do not include possible bounds from such searches and, instead we point unique features of light, lepton-flavor violating mediators that may pose challenges and opportunities for future experiments. The range of dark photon/light mediator experiments are mapped out in recent white papers on this subject, refs. [138, 139]. Of the menagerie of such experiments, the lepton-flavor violating mediator examined here is only potentially visible in a subset, which require some modifications.

The first requirement is that the experiment must involve leptons in order to couple to $\varphi$. Thus one is restricted to experiments with an electron beam. Because of the flavor violating couplings, $e^{+} e^{-}$annihilation to $\varphi$ would occur through $\varphi$ pair production. This is suppressed by multiple small couplings and one cannot leverage the bump hunt strategy in $e^{+} e^{-} \rightarrow \gamma A^{\prime}$. Instead, one is led to fixed target experiments that invoke $\varphi$ bremsstrahlung, $e^{-} N \rightarrow \mu^{-} N \varphi$; where $N$ is a heavy target nucleus. This is shown in figure $7 \mathrm{~b}$. Observe that this differs from the case of a dark photon, $e^{-} N \rightarrow e^{-} N A^{\prime}$, in that a muon is produced in association with the $\varphi$ as well as in the $\varphi$ decay. These searches, then, not only require higher energy electron beams, but may also have very different kinematics from the dark photon case in ref. [140]. One must also be careful that the searches for visible decays of a dark photon are sensitive to muons: since muons are minimum-ionizing at the typical energy scales of these experiments, one must confirm that the detectors have reasonable muon energy and/or vertex reconstruction. 
For these reasons, we leave the re-interpretation of dark photon experiments to the search for lepton-flavor violating mediators for separate work. We feel that this is an interesting experimental question and may be a fruitful way to extend our search for light, weakly-coupled new physics. To the best of our knowledge, none of the experiments listed in ref. [139] has a search for a lepton-flavor violating mediator of the type discussed here. The proposed "SuperHPS" experiment may be sensitive to $\varphi$ bremsstrahlung if the kinematics, masses, and displaced vertex resolution is amenable [139]. We note that the SeaQuest experiment is unique in that it features the ability to accurately reconstruct muons; however, the proton initial state makes it difficult to produce the mediator [141].

\subsection{Collider searches}

Standard proton collider searches are limited in their reach to search for a lepton-flavor violating mediator due to the electroweak couplings required to produce progenitor leptons which may produce the mediator. That being said, these mediators can produce striking experimental signatures that are difficult to fake in the Standard Model. In this case, it is useful to invoke the electroweak-scale, non-renormalizable Lagrangian interaction (2.5) that was assumed to generate the low-energy couplings (2.4). From this interaction, one can consider Higgs decays such as $h \rightarrow \varphi^{*} \mu \bar{e} \rightarrow \mu \bar{\mu} e \bar{e}$. This is shown in figure 7c. Note that with the chiral structure imposed, one cannot produce Higgs decays to two pairs of samesign, same-flavor leptons unless $\varphi$ is a real field. The possibility of an exotic flavor violating Higgs decay mediated by new scalars has recently generated attention [142-144] in part due to the possibility of an observation of $h \rightarrow \tau \mu$ at the Large Hadron Collider [61, 62], though those claims appear to be in tension with early results from Run II [145, 146]. We postpone a discussion to future work [147].

While the ideal collider search would require a muon-electron collider [148], low-energy $e^{+} e^{-}$colliders like the $B$-factories may be sensitive to lepton-flavor violating mediators. The upcoming Belle-II run, for example, will run at $\sqrt{s}=10 \mathrm{GeV}$ and is sensitive to muon final states [149]. The symmetry structure dictates that if $\varphi$ s are produced, the leading signals involve an even number of opposite-sign same-flavor lepton pairs. For example, one may search for $e^{+} e^{-} \rightarrow e \bar{e} \mu \bar{\mu}$. This process has contributions from $\varphi \varphi^{*}$ pair production, in which each opposite-flavor-opposite-charge pair reconstructs a $\varphi$, as shown in figure $7 \mathrm{~d}$, or at higher order through a flavor changing $\varphi$-strahlung off an initial $e^{+}$state. Similar symmetry arguments apply for dark matter searches in possible future high-energy $e^{+} e^{-}$colliders. In this case, the production of dark matter is $\varphi$-mediated, so the leading contributions are: (a) $e^{+} e^{-} \rightarrow \chi \bar{\chi} \chi \bar{\chi}$, which is suppressed at order $g_{e \mu}^{4}$ and its reach is limited by phase-space due to the multiple dark matter particles, and (b) $e^{+} e^{-} \rightarrow \chi \bar{\chi} \varphi, \varphi \rightarrow e \mu$. which is also $g_{e \mu}^{4}$ suppressed.

\section{Conclusions \& outlook}

In this manuscript we examined a class of models where dark matter interacts with the Standard Model through spin-0 mediators with chiral, flavor violating interactions to leptons. For a range of mediator masses, this set up realizes the secluded dark matter scenario 


\begin{tabular}{|llllll|}
\hline Dark parity of $\varphi$ & Annihlation & $\ell_{i} \ell_{j}$ & $m_{\chi} / \mathrm{GeV}$ & $\frac{\langle\sigma v\rangle}{\langle\sigma v\rangle_{\text {rel. }}}$ & AMS-02 \\
\hline Not parity eigenstate & $\tau e$ & $20-40$ & $1(0.4)$ & $\checkmark$ \\
& & $\tau \mu$ & $20-40$ & $1(0.4)$ & $\checkmark$ \\
Pseudoscalar (parity-odd) & & $\tau e$ & $30-90$ & $3(1.1)$ & $\times(\checkmark)$ \\
\hline
\end{tabular}

Table 1. Summary of figures 2 and 3. The annihilation cross-section, $\langle\sigma v\rangle$ is given for $\gamma_{\mathrm{NFW}}=1.0$ (1.2). The $\mu$ e modes are in tension with the AMS-02 positron bound unless one takes the contracted $\gamma_{\mathrm{NFW}}=1.2$ profile. The range of mediator masses are given in (2.3).

where the relic abundance and indirect detection annihilation rates are controlled by one set of couplings, while direct detection, collider bounds, and low-energy searches are controlled by a separate set of couplings.

We have shown that in the dark sector, one is able to simultaneously achieve a thermal relic and the observed Fermi $\gamma$-ray excess without causing tension with the measured AMS-02 positron spectrum. The $\gamma$-ray excess is produced through a combination of prompt photon emission for $\tau$ final states and the inverse Compton scattering of the interstellar radiation field for $\mu$ and $e$ final states. Because dwarf spheroidals have much weaker interstellar radiation fields, this helps alleviate tensions of the $\gamma$-ray excess with non-observations in dwarf spheroidals. For the case of $\mu$ interactions, however, this requires a contracted dark matter halo profile. The spectra of the Standard Model byproducts of dark matter annihilation are softened because the decay goes through on-shell mediators. This smearing helps the $e^{+} e^{-}$spectrum to fit within the error bars of the AMS experiment. We have commented that the parameters for the $\mu e$ final state appear to be consistent with the target region for a self-interacting dark matter solution to small scale structure anomalies. The dark sector interactions are summarized in table 1.

One unique feature of the chiral lepton-flavor violating interactions is that the bounds on the Standard Model couplings are weaker than direct flavor-conserving interactions. We have shown that the upper bounds on the Standard Model interactions in this scenario come from the forward-backward asymmetry in $e^{+} e^{-} \rightarrow f \bar{f}$ and from the anomalous magnetic moments of the muon and electron. For the case of a $\tau \mu$ interaction, one can simultaneously explain the $(g-2)_{\mu}$ anomaly. We have shown that the chiral flavor structure of the mediator-Standard Model interaction suppresses bounds from conventional charged lepton flavor violation experiments and direct detection experiments. We explained that these suppressions are straightforward to understand from the point of view of a spurious $L_{i}-L_{j}$ symmetry that is respected by the mediator when it is complex. Finally, we point out possible opportunities in dark photon experiments and collider searches that are motivated by this class of mediator models. 
We emphasize that while we have benchmarked our models for the Fermi $\gamma$-ray excess, the class of models are independently meaningful as an example of light, weakly-coupled new physics that can play an important role in both Standard Model and dark matter phenomenology and that are able to avoid current constraints.

\section{Acknowledgments}

We enthusiastically thank Sheldon Campbell, Jared Evans, Paddy Fox, Manoj Kaplinghat, Ranjan Laha, Gørdån Krnjåiç, Nirmal Raj, Arvind Rajaraman, Yael Shadmi, Tim M. P. Tait, Scott Thomas, Hai-Bo Yu, and Yue Zhao for many useful comments and discussions. We especially thank Jared for discussions regarding the contribution to anomalous magnetic moments and possible collider searches at high luminosity, Paddy for pointing out the possible relation to $h \rightarrow \tau \mu$, and Yael for suggesting that a complex mediator can be treated as a spurion for $L_{i}-L_{j}$ breaking. A.K. is supported by an NSF graduate research fellowship. P.T. thanks the Aspen Center for Physics (NSF grant \#1066293) for its hospitality during a period where part of this work was completed and sends hugs to Jane Kelly and Patty Fox. This work is supported in part by the NSF grant PHY-1316792 and NSF grant-1620638.

Open Access. This article is distributed under the terms of the Creative Commons Attribution License (CC-BY 4.0), which permits any use, distribution and reproduction in any medium, provided the original author(s) and source are credited.

\section{References}

[1] S. Arrenberg et al., Working group report: dark matter complementarity, in Community Summer Study 2013: Snowmass on the Mississippi (Cs $\left.S^{2} 013\right)$, July 29-August 6, Minneapolis, U.S.A. (2013), arXiv:1310.8621 [INSPIRE].

[2] M. Pospelov, A. Ritz and M.B. Voloshin, Secluded WIMP dark matter, Phys. Lett. B 662 (2008) 53 [arXiv:0711.4866] [INSPIRE].

[3] L. Goodenough and D. Hooper, Possible evidence for dark matter annihilation in the inner Milky Way from the Fermi Gamma Ray Space Telescope, arXiv:0910.2998 [InSPIRE].

[4] D. Hooper and L. Goodenough, Dark matter annihilation in the Galactic Center as seen by the Fermi Gamma Ray Space Telescope, Phys. Lett. B 697 (2011) 412 [arXiv:1010.2752] [INSPIRE].

[5] K.N. Abazajian, The consistency of Fermi-LAT observations of the Galactic Center with a millisecond pulsar population in the central stellar cluster, JCAP 03 (2011) 010 [arXiv: 1011.4275] [INSPIRE].

[6] A. Boyarsky, D. Malyshev and O. Ruchayskiy, A comment on the emission from the Galactic Center as seen by the Fermi telescope, Phys. Lett. B 705 (2011) 165 [arXiv: 1012.5839] [INSPIRE].

[7] D. Hooper and T. Linden, On the origin of the gamma rays from the Galactic Center, Phys. Rev. D 84 (2011) 123005 [arXiv:1110.0006] [INSPIRE]. 
[8] K.N. Abazajian and M. Kaplinghat, Detection of a gamma-ray source in the galactic center consistent with extended emission from dark matter annihilation and concentrated astrophysical emission, Phys. Rev. D 86 (2012) 083511 [Erratum ibid. D 87 (2013) 129902] [arXiv: 1207.6047] [INSPIRE].

[9] C. Gordon and O. Macias, Dark matter and pulsar model constraints from galactic center Fermi-LAT gamma ray observations, Phys. Rev. D 88 (2013) 083521 [arXiv:1306.5725] [INSPIRE].

[10] D. Hooper and T.R. Slatyer, Two emission mechanisms in the Fermi bubbles: a possible signal of annihilating dark matter, Phys. Dark Univ. 2 (2013) 118 [arXiv:1302.6589] [INSPIRE].

[11] W.-C. Huang, A. Urbano and W. Xue, Fermi bubbles under dark matter scrutiny. Part I: astrophysical analysis, arXiv: 1307.6862 [INSPIRE].

[12] N. Okada and O. Seto, Gamma ray emission in Fermi bubbles and Higgs portal dark matter, Phys. Rev. D 89 (2014) 043525 [arXiv:1310.5991] [INSPIRE].

[13] O. Macias and C. Gordon, Contribution of cosmic rays interacting with molecular clouds to the Galactic Center gamma-ray excess, Phys. Rev. D 89 (2014) 063515 [arXiv:1312.6671] [INSPIRE].

[14] K.N. Abazajian, N. Canac, S. Horiuchi and M. Kaplinghat, Astrophysical and dark matter interpretations of extended gamma-ray emission from the Galactic Center, Phys. Rev. D 90 (2014) 023526 [arXiv: 1402.4090] [INSPIRE].

[15] T. Daylan et al., The characterization of the gamma-ray signal from the central Milky Way: a case for annihilating dark matter, Phys. Dark Univ. 12 (2016) 1 [arXiv:1402.6703] [INSPIRE].

[16] B. Zhou et al., GeV excess in the Milky Way: the role of diffuse galactic gamma-ray emission templates, Phys. Rev. D 91 (2015) 123010 [arXiv:1406.6948] [INSPIRE].

[17] F. Calore, I. Cholis and C. Weniger, Background model systematics for the Fermi GeV excess, JCAP 03 (2015) 038 [arXiv:1409.0042] [INSPIRE].

[18] F. Calore, I. Cholis, C. McCabe and C. Weniger, A tale of tails: dark matter interpretations of the Fermi GeV excess in light of background model systematics, Phys. Rev. D 91 (2015) 063003 [arXiv: 1411.4647] [INSPIRE].

[19] F. Calore, I. Cholis and C. Weniger, The GeV excess shining through: background systematics for the inner galaxy analysis, arXiv:1502.02805 [INSPIRE].

[20] Fermi-LAT collaboration, M. Ajello et al., Fermi-LAT observations of high-energy $\gamma$-ray emission toward the Galactic Center, Astrophys. J. 819 (2016) 44 [arXiv:1511.02938] [INSPIRE].

[21] D. Hooper, I. Cholis, T. Linden, J. Siegal-Gaskins and T. Slatyer, Pulsars cannot account for the inner galaxy's GeV excess, Phys. Rev. D 88 (2013) 083009 [arXiv:1305.0830] [INSPIRE].

[22] Q. Yuan and B. Zhang, Millisecond pulsar interpretation of the Galactic center gamma-ray excess, JHEAp 3-4 (2014) 1 [arXiv: 1404.2318] [INSPIRE].

[23] J. Petrović, P.D. Serpico and G. Zaharijas, Millisecond pulsars and the Galactic Center gamma-ray excess: the importance of luminosity function and secondary emission, JCAP 02 (2015) 023 [arXiv: 1411.2980] [INSPIRE]. 
[24] R. Bartels, S. Krishnamurthy and C. Weniger, Strong support for the millisecond pulsar origin of the Galactic center GeV excess, Phys. Rev. Lett. 116 (2016) 051102 [arXiv: 1506.05104] [INSPIRE].

[25] S.K. Lee, M. Lisanti, B.R. Safdi, T.R. Slatyer and W. Xue, Evidence for unresolved $\gamma$-ray point sources in the inner galaxy, Phys. Rev. Lett. 116 (2016) 051103 [arXiv:1506.05124] [INSPIRE].

[26] S.D. McDermott, P.J. Fox, I. Cholis and S.K. Lee, Wavelet-based techniques for the gamma-ray sky, JCAP 07 (2016) 045 [arXiv: 1512.00012] [INSPIRE].

[27] D. Hooper and G. Mohlabeng, The gamma-ray luminosity function of millisecond pulsars and implications for the GeV excess, JCAP 03 (2016) 049 [arXiv: 1512.04966] [INSPIRE].

[28] J. Petrović, P.D. Serpico and G. Zaharijaš, Galactic Center gamma-ray "excess" from an active past of the Galactic Centre?, JCAP 10 (2014) 052 [arXiv:1405.7928] [INSPIRE].

[29] E. Carlson and S. Profumo, Cosmic ray protons in the inner galaxy and the Galactic Center gamma-ray excess, Phys. Rev. D 90 (2014) 023015 [arXiv:1405.7685] [INSPIRE].

[30] E. Carlson and S. Profumo, When dark matter interacts with cosmic rays or interstellar matter: a morphological study, Phys. Rev. D 92 (2015) 063003 [arXiv: 1504.04782] [INSPIRE].

[31] I. Cholis, C. Evoli, F. Calore, T. Linden, C. Weniger and D. Hooper, The galactic center GeV excess from a series of leptonic cosmic-ray outbursts, JCAP 12 (2015) 005 [arXiv: 1506. 05119] [INSPIRE].

[32] C. Boehm, M.J. Dolan, C. McCabe, M. Spannowsky and C.J. Wallace, Extended gamma-ray emission from Coy dark matter, JCAP 05 (2014) 009 [arXiv:1401.6458] [INSPIRE].

[33] A. Alves, S. Profumo, F.S. Queiroz and W. Shepherd, Effective field theory approach to the Galactic Center gamma-ray excess, Phys. Rev. D 90 (2014) 115003 [arXiv:1403.5027] [INSPIRE].

[34] S. Ipek, D. McKeen and A.E. Nelson, A renormalizable model for the Galactic Center gamma ray excess from dark matter annihilation, Phys. Rev. D 90 (2014) 055021 [arXiv:1404.3716] [INSPIRE].

[35] E. Izaguirre, G. Krnjaic and B. Shuve, The Galactic Center excess from the bottom up, Phys. Rev. D 90 (2014) 055002 [arXiv: 1404.2018] [INSPIRE].

[36] A. Berlin, D. Hooper and S.D. McDermott, Simplified dark matter models for the Galactic Center gamma-ray excess, Phys. Rev. D 89 (2014) 115022 [arXiv:1404.0022] [INSPIRE].

[37] P. Agrawal, B. Batell, P.J. Fox and R. Harnik, WIMPs at the Galactic Center, JCAP 05 (2015) 011 [arXiv: 1411.2592] [INSPIRE].

[38] C. Boehm, M.J. Dolan and C. McCabe, A weighty interpretation of the Galactic Centre excess, Phys. Rev. D 90 (2014) 023531 [arXiv:1404.4977] [InSPIRE].

[39] M. Abdullah et al., Hidden on-shell mediators for the Galactic Center $\gamma$-ray excess, Phys. Rev. D 90 (2014) 035004 [arXiv: 1404.6528] [INSPIRE].

[40] A. Martin, J. Shelton and J. Unwin, Fitting the Galactic Center gamma-ray excess with cascade annihilations, Phys. Rev. D 90 (2014) 103513 [arXiv:1405.0272] [INSPIRE].

[41] A. Rajaraman, J. Smolinsky and P. Tanedo, On-shell mediators and top-charm dark matter models for the Fermi-LAT Galactic Center excess, arXiv:1503.05919 [INSPIRE].

[42] G. Elor, N.L. Rodd and T.R. Slatyer, Multistep cascade annihilations of dark matter and the Galactic Center excess, Phys. Rev. D 91 (2015) 103531 [arXiv:1503.01773] [INSPIRE]. 
[43] K.N. Abazajian and R.E. Keeley, Bright gamma-ray Galactic Center excess and dark dwarfs: Strong tension for dark matter annihilation despite Milky Way halo profile and diffuse emission uncertainties, Phys. Rev. D 93 (2016) 083514 [arXiv:1510.06424] [INSPIRE].

[44] DES, Fermi-LAT collaboration, A. Drlica-Wagner et al., Search for gamma-ray emission from DES dwarf spheroidal galaxy candidates with Fermi-LAT data, Astrophys. J. 809 (2015) L4 [arXiv:1503.02632] [INSPIRE].

[45] Fermi-LAT collaboration, M. Ackermann et al., Searching for dark matter annihilation from Milky Way dwarf spheroidal galaxies with six years of Fermi Large Area Telescope data, Phys. Rev. Lett. 115 (2015) 231301 [arXiv:1503.02641] [INSPIRE].

[46] A. Geringer-Sameth et al., Indication of gamma-ray emission from the newly discovered dwarf galaxy Reticulum II, Phys. Rev. Lett. 115 (2015) 081101 [arXiv:1503.02320] [inSPIRE].

[47] D. Hooper and T. Linden, On the gamma-ray emission from Reticulum II and other dwarf galaxies, JCAP 09 (2015) 016 [arXiv: 1503.06209] [INSPIRE].

[48] S. Li et al., Search for gamma-ray emission from eight dwarf spheroidal galaxy candidates discovered in Year Two of Dark Energy Survey with Fermi-LAT data, Phys. Rev. D 93 (2016) 043518 [arXiv : 1511.09252] [inSPIRE].

[49] T. Lacroix, C. Boehm and J. Silk, Fitting the Fermi-LAT GeV excess: on the importance of including the propagation of electrons from dark matter, Phys. Rev. D 90 (2014) 043508 [arXiv: 1403.1987] [INSPIRE].

[50] M. Kaplinghat, T. Linden and H.-B. Yu, Galactic center excess in $\gamma$ rays from annihilation of self-interacting dark matter, Phys. Rev. Lett. 114 (2015) 211303 [arXiv:1501.03507] [INSPIRE].

[51] S. Tulin, H.-B. Yu and K.M. Zurek, Beyond collisionless dark matter: particle physics dynamics for dark matter halo structure, Phys. Rev. D 87 (2013) 115007 [arXiv:1302.3898] [INSPIRE].

[52] M. Kaplinghat, S. Tulin and H.-B. Yu, Dark matter halos as particle colliders: unified solution to small-scale structure puzzles from dwarfs to clusters, Phys. Rev. Lett. 116 (2016) 041302 [arXiv: 1508.03339] [INSPIRE].

[53] C.D. Froggatt and H.B. Nielsen, Hierarchy of quark masses, Cabibbo angles and CP-violation, Nucl. Phys. B 147 (1979) 277 [inSPIRE].

[54] J. Heeck, Lepton flavor violation with light vector bosons, Phys. Lett. B 758 (2016) 101 [arXiv:1602.03810] [INSPIRE].

[55] W. Altmannshofer, C.-Y. Chen, P.S. Bhupal Dev and A. Soni, Lepton flavor violating $Z^{\prime}$ explanation of the muon anomalous magnetic moment, Phys. Lett. B 762 (2016) 389 [arXiv: 1607.06832] [INSPIRE].

[56] LHCb collaboration, Test of lepton universality using $B^{+} \rightarrow K^{+} \ell^{+} \ell^{-}$decays, Phys. Rev. Lett. 113 (2014) 151601 [arXiv: 1406.6482] [INSPIRE].

[57] BABAR collaboration, J.P. Lees et al., Evidence for an excess of $\bar{B} \rightarrow D^{(*)} \tau^{-} \bar{\nu}_{\tau}$ decays, Phys. Rev. Lett. 109 (2012) 101802 [arXiv:1205.5442] [INSPIRE].

[58] BELLE collaboration, M. Huschle et al., Measurement of the branching ratio of $\bar{B} \rightarrow D^{(*)} \tau^{-} \bar{\nu}_{\tau}$ relative to $\bar{B} \rightarrow D^{(*)} \ell^{-} \bar{\nu}_{\ell}$ decays with hadronic tagging at Belle, Phys. Rev. D 92 (2015) 072014 [arXiv:1507.03233] [INSPIRE]. 
[59] BELle collaboration, A. Abdesselam et al., Measurement of the branching ratio of $\bar{B}^{0} \rightarrow D^{*+} \tau^{-} \bar{\nu}_{\tau}$ relative to $\bar{B}^{0} \rightarrow D^{*+} \ell^{-} \bar{\nu}_{\ell}$ decays with a semileptonic tagging method, arXiv: 1603.06711 [INSPIRE].

[60] LHCb collaboration, Measurement of the ratio of branching fractions $\mathcal{B}\left(\bar{B}^{0} \rightarrow D^{*+} \tau^{-} \bar{\nu}_{\tau}\right) / \mathcal{B}\left(\bar{B}^{0} \rightarrow D^{*+} \mu^{-} \bar{\nu}_{\mu}\right)$, Phys. Rev. Lett. 115 (2015) 111803 [arXiv: 1506.08614] [INSPIRE].

[61] CMS collaboration, Search for lepton-flavour-violating decays of the Higgs boson, Phys. Lett. B 749 (2015) 337 [arXiv: 1502.07400] [INSPIRE].

[62] ATLAS collaboration, Search for lepton-flavour-violating $H \rightarrow \mu \tau$ decays of the Higgs boson with the ATLAS detector, JHEP 11 (2015) 211 [arXiv:1508.03372] [INSPIRE].

[63] J. Kile and A. Soni, Flavored dark matter in direct detection experiments and at LHC, Phys. Rev. D 84 (2011) 035016 [arXiv: 1104.5239] [InSPIRE].

[64] B. Batell, J. Pradler and M. Spannowsky, Dark matter from minimal flavor violation, JHEP 08 (2011) 038 [arXiv:1105.1781] [INSPIRE].

[65] J.F. Kamenik and J. Zupan, Discovering dark matter through flavor violation at the LHC, Phys. Rev. D 84 (2011) 111502 [arXiv:1107.0623] [INSPIRE].

[66] P. Agrawal, S. Blanchet, Z. Chacko and C. Kilic, Flavored dark matter and its implications for direct detection and colliders, Phys. Rev. D 86 (2012) 055002 [arXiv:1109.3516] [INSPIRE].

[67] L. Lopez-Honorez and L. Merlo, Dark matter within the minimal flavour violation ansatz, Phys. Lett. B 722 (2013) 135 [arXiv:1303.1087] [INSPIRE].

[68] B. Batell, T. Lin and L.-T. Wang, Flavored dark matter and R-parity violation, JHEP 01 (2014) 075 [arXiv: 1309.4462] [INSPIRE].

[69] J. Kile, Flavored dark matter: a review, Mod. Phys. Lett. A 28 (2013) 1330031 [arXiv:1308.0584] [INSPIRE].

[70] A. Kumar and S. Tulin, Top-flavored dark matter and the forward-backward asymmetry, Phys. Rev. D 87 (2013) 095006 [arXiv: 1303.0332] [InSPIRE].

[71] P. Agrawal, B. Batell, D. Hooper and T. Lin, Flavored dark matter and the Galactic Center gamma-ray excess, Phys. Rev. D 90 (2014) 063512 [arXiv:1404.1373] [INSPIRE].

[72] P. Agrawal, M. Blanke and K. Gemmler, Flavored dark matter beyond minimal flavor violation, JHEP 10 (2014) 72 [arXiv:1405.6709] [INSPIRE].

[73] J. D'Hondt, A. Mariotti, K. Mawatari, S. Moortgat, P. Tziveloglou and G. Van Onsem, Signatures of top flavour-changing dark matter, JHEP 03 (2016) 060 [arXiv:1511.07463] [INSPIRE].

[74] P. Agrawal, Z. Chacko, E.C. F.S. Fortes and C. Kilic, Skew-flavored dark matter, Phys. Rev. D 93 (2016) 103510 [arXiv: 1511.06293] [InSPIRE].

[75] M.-C. Chen, J. Huang and V. Takhistov, Beyond minimal lepton flavored dark matter, JHEP 02 (2016) 060 [arXiv:1510.04694] [InSPIRE].

[76] B. Bhattacharya, D. London, J.M. Cline, A. Datta and G. Dupuis, Quark-flavored scalar dark matter, Phys. Rev. D 92 (2015) 115012 [arXiv:1509.04271] [INSPIRE].

[77] F. Bishara, A. Greljo, J.F. Kamenik, E. Stamou and J. Zupan, Dark matter and gauged flavor symmetries, JHEP 12 (2015) 130 [arXiv:1505.03862] [INSPIRE].

[78] P. Agrawal, Z. Chacko, C. Kilic and C.B. Verhaaren, A couplet from flavored dark matter, JHEP 08 (2015) 072 [arXiv: 1503.03057] [INSPIRE]. 
[79] U. Haisch and E. Re, Simplified dark matter top-quark interactions at the LHC, JHEP 06 (2015) 078 [arXiv:1503.00691] [INSPIRE].

[80] L. Calibbi, A. Crivellin and B. Zaldívar, Flavor portal to dark matter, Phys. Rev. D 92 (2015) 016004 [arXiv: 1501.07268] [InSPIRE].

[81] C. Kilic, M.D. Klimek and J.-H. Yu, Signatures of top flavored dark matter, Phys. Rev. D 91 (2015) 054036 [arXiv: 1501.02202] [InSPIRE].

[82] M.A. Fedderke, J.-Y. Chen, E.W. Kolb and L.-T. Wang, The fermionic dark matter Higgs portal: an effective field theory approach, JHEP 08 (2014) 122 [arXiv: 1404.2283] [INSPIRE].

[83] G. Steigman, B. Dasgupta and J.F. Beacom, Precise relic WIMP abundance and its impact on searches for dark matter annihilation, Phys. Rev. D 86 (2012) 023506 [arXiv:1204.3622] [INSPIRE].

[84] J.M. Cline, G. Dupuis, Z. Liu and W. Xue, Multimediator models for the galactic center gamma ray excess, Phys. Rev. D 91 (2015) 115010 [arXiv:1503.08213] [INSPIRE].

[85] ARGUS collaboration, H. Albrecht et al., A Search for lepton flavor violating decays $\tau \rightarrow e \alpha, \tau \rightarrow \mu \alpha, Z$. Phys. C 68 (1995) 25 [INSPIRE].

[86] TWIST collaboration, R. Bayes et al., Search for two body muon decay signals, Phys. Rev. D 91 (2015) 052020 [arXiv:1409.0638] [INSPIRE].

[87] A. Halprin and A. Masiero, Muonium-anti-muonium oscillations and exotic muon decay in broken R-parity SUSY models, Phys. Rev. D 48 (1993) R2987.

[88] R.N. Mohapatra, Rare muon decays and physics beyond the standard model, Z. Phys. C 56 (1992) S117.

[89] M. Leurer, Y. Nir and N. Seiberg, Mass matrix models, Nucl. Phys. B 398 (1993) 319 [hep-ph/9212278] [INSPIRE].

[90] Y. Nir and N. Seiberg, Should squarks be degenerate?, Phys. Lett. B 309 (1993) 337 [hep-ph/9304307] [INSPIRE].

[91] M. Leurer, Y. Nir and N. Seiberg, Mass matrix models: the sequel, Nucl. Phys. B 420 (1994) 468 [hep-ph/9310320] [INSPIRE].

[92] Y. Shadmi and P.Z. Szabo, Flavored gauge-mediation, JHEP 06 (2012) 124 [arXiv:1103.0292] [INSPIRE].

[93] M. Abdullah, I. Galon, Y. Shadmi and Y. Shirman, Flavored gauge mediation, a heavy Higgs and supersymmetric alignment, JHEP 06 (2013) 057 [arXiv: 1209.4904] [INSPIRE].

[94] I. Galon, G. Perez and Y. Shadmi, Non-degenerate squarks from flavored gauge mediation, JHEP 09 (2013) 117 [arXiv:1306.6631] [INSPIRE].

[95] D. Harvey, R. Massey, T. Kitching, A. Taylor and E. Tittley, The non-gravitational interactions of dark matter in colliding galaxy clusters, Science 347 (2015) 1462 [arXiv: 1503.07675] [INSPIRE].

[96] J.L. Feng, H. Tu and H.-B. Yu, Thermal relics in hidden sectors, JCAP 10 (2008) 043 [arXiv: 0808.2318] [INSPIRE].

[97] B. Bellazzini, M. Cliche and P. Tanedo, Effective theory of self-interacting dark matter, Phys. Rev. D 88 (2013) 083506 [arXiv:1307.1129] [InSPIRE].

[98] M. Cirelli et al., PPPC \& DM ID: a poor particle physicist cookbook for dark matter indirect detection, JCAP 03 (2011) 051 [Erratum ibid. 10 (2012) E01] [arXiv:1012.4515] [INSPIRE]. 
[99] J. Buch, M. Cirelli, G. Giesen and M. Taoso, PPPC \& DM secondary: a poor particle physicist cookbook for secondary radiation from dark matter, JCAP 09 (2015) 037 [arXiv: 1505.01049] [INSPIRE].

[100] J. Mardon, Y. Nomura, D. Stolarski and J. Thaler, Dark matter signals from cascade annihilations, JCAP 05 (2009) 016 [arXiv:0901.2926] [INSPIRE].

[101] T. Sjöstrand, S. Mrenna and P.Z. Skands, PYTHIA 6.4 physics and manual, JHEP 05 (2006) 026 [hep-ph/0603175] [INSPIRE].

[102] T. Delahaye, R. Lineros, F. Donato, N. Fornengo and P. Salati, Positrons from dark matter annihilation in the galactic halo: Theoretical uncertainties, Phys. Rev. D 77 (2008) 063527 [arXiv:0712.2312] [INSPIRE].

[103] P. Salucci, F. Nesti, G. Gentile and C.F. Martins, The dark matter density at the Sun's location, Astron. Astrophys. 523 (2010) A83 [arXiv:1003.3101] [INSPIRE].

[104] S. Horiuchi, M. Kaplinghat and A. Kwa, Investigating the uniformity of the excess gamma rays towards the Galactic Center region, JCAP 11 (2016) 053 [arXiv:1604.01402] [INSPIRE].

[105] L. Bergstrom, T. Bringmann, I. Cholis, D. Hooper and C. Weniger, New limits on dark matter annihilation from AMS cosmic ray positron data, Phys. Rev. Lett. 111 (2013) 171101 [arXiv: 1306 . 3983] [INSPIRE].

[106] AMS collaboration, M. Aguilar et al., Electron and positron fluxes in primary cosmic rays measured with the alpha magnetic spectrometer on the international space station, Phys. Rev. Lett. 113 (2014) 121102 [INSPIRE].

[107] D. Gaggero, L. Maccione, G. Di Bernardo, C. Evoli and D. Grasso, Three-dimensional model of cosmic-ray lepton propagation reproduces data from the alpha magnetic spectrometer on the International Space Station, Phys. Rev. Lett. 111 (2013) 021102 [arXiv:1304.6718] [INSPIRE].

[108] K.N. Abazajian, P. Agrawal, Z. Chacko and C. Kilic, Lower limits on the strengths of gamma ray lines from WIMP dark matter annihilation, Phys. Rev. D 85 (2012) 123543 [arXiv:1111.2835] [INSPIRE].

[109] P. Langacker, Precision tests of standard model of electroweak interactions, World Scientific, Singapore (1996).

[110] V.D. Barger, G.F. Giudice and T. Han, Some new aspects of supersymmetry R-parity violating interactions, Phys. Rev. D 40 (1989) 2987 [INSPIRE].

[111] R. Barbier et al., R-parity violating supersymmetry, Phys. Rept. 420 (2005) 1 [hep-ph/0406039] [INSPIRE].

[112] SLD Electroweak Group, DelPhi, AlePh, SLD, SLD Heavy Flavour Group, OPAL, LEP Electroweak Working Group, L3 collaboration, S. Schael et al., Precision electroweak measurements on the $Z$ resonance, Phys. Rept. 427 (2006) 257 [hep-ex/0509008] [inSPIRE].

[113] R. Laha, B. Dasgupta and J.F. Beacom, Constraints on new neutrino interactions via light abelian vector bosons, Phys. Rev. D 89 (2014) 093025 [arXiv:1304.3460] [INSPIRE].

[114] M.E. Peskin and T. Takeuchi, Estimation of oblique electroweak corrections, Phys. Rev. D 46 (1992) 381 [INSPIRE].

[115] M. Pospelov and A. Ritz, Electric dipole moments as probes of new physics, Annals Phys. 318 (2005) 119 [hep-ph/0504231] [INSPIRE]. 
[116] M. Pospelov, Secluded U(1) below the weak scale, Phys. Rev. D 80 (2009) 095002 [arXiv:0811.1030] [INSPIRE].

[117] H. Davoudiasl, H.-S. Lee and W.J. Marciano, Dark side of Higgs diphoton decays and muon $g-2$, Phys. Rev. D 86 (2012) 095009 [arXiv: 1208.2973] [INSPIRE].

[118] M. Endo, K. Hamaguchi and G. Mishima, Constraints on hidden photon models from electron $g-2$ and hydrogen spectroscopy, Phys. Rev. D 86 (2012) 095029 [arXiv: 1209.2558] [INSPIRE].

[119] G.F. Giudice, P. Paradisi and M. Passera, Testing new physics with the electron $g-2$, JHEP 11 (2012) 113 [arXiv:1208.6583] [InSPIRE].

[120] M. Lindner, M. Platscher and F.S. Queiroz, A call for new physics: the muon anomalous magnetic moment and lepton flavor violation, arXiv:1610.06587 [INSPIRE].

[121] J.E. Kim, B. Kyae and H.M. Lee, Effective supersymmetric theory and $(g-2)$ (muon) with R-parity violation, Phys. Lett. B 520 (2001) 298 [hep-ph/0103054] [INSPIRE].

[122] D. Hanneke, S. Fogwell and G. Gabrielse, New measurement of the electron magnetic moment and the fine structure constant, Phys. Rev. Lett. 100 (2008) 120801 [arXiv:0801.1134] [INSPIRE].

[123] T. Aoyama, M. Hayakawa, T. Kinoshita and M. Nio, Tenth-order QED contribution to the electron $g-2$ and an improved value of the fine structure constant, Phys. Rev. Lett. 109 (2012) 111807 [arXiv:1205.5368] [INSPIRE].

[124] Muon G-2 collaboration, G.W. Bennett et al., Final report of the muon E821 anomalous magnetic moment measurement at BNL, Phys. Rev. D 73 (2006) 072003 [hep-ex/0602035] [INSPIRE].

[125] H. Davoudiasl, H.S. Lee and W.J. Marciano, Muon $g-2$, rare kaon decays and parity violation from dark bosons, Phys. Rev. D 89 (2014) 095006 [arXiv: 1402.3620] [INSPIRE].

[126] R.H. Bernstein and P.S. Cooper, Charged lepton flavor violation: an experimenter's guide, Phys. Rept. 532 (2013) 27 [arXiv: 1307.5787] [INSPIRE].

[127] MEG collaboration, J. Adam et al., New constraint on the existence of the $\mu^{+} \rightarrow e^{+} \gamma$ decay, Phys. Rev. Lett. 110 (2013) 201801 [arXiv:1303.0754] [InSPIRE].

[128] BABAR collaboration, B. Aubert et al., Searches for lepton flavor violation in the decays $\tau^{ \pm} \rightarrow e^{ \pm} \gamma$ and $\tau^{ \pm} \rightarrow \mu^{ \pm} \gamma$, Phys. Rev. Lett. 104 (2010) 021802 [arXiv:0908.2381] [INSPIRE].

[129] SINDRUM II collaboration, W.H. Bertl et al., A search for muon to electron conversion in muonic gold, Eur. Phys. J. C 47 (2006) 337 [INSPIRE].

[130] SINDRUM II collaboration, C. Dohmen et al., Test of lepton flavor conservation in $\mu \rightarrow e$ conversion on titanium, Phys. Lett. B 317 (1993) 631 [INSPIRE].

[131] Mu2E collaboration, R.J. Abrams et al., Mu2e conceptual design report, arXiv:1211.7019 [INSPIRE].

[132] SINDRUM collaboration, U. Bellgardt et al., Search for the decay $\mu^{+} \rightarrow e^{+} e^{+} e^{-}$, Nucl. Phys. B 299 (1988) 1 [inSPIRE].

[133] K. Hayasaka et al., Search for lepton flavor violating $\tau$ decays into three leptons with 719 million produced $\tau^{+} \tau^{-}$pairs, Phys. Lett. B 687 (2010) 139 [arXiv:1001.3221] [INSPIRE].

[134] SINDRUM collaboration, W.H. Bertl et al., Search for the decay $\mu^{+} \rightarrow e^{+} e^{+} e^{-}$, Nucl. Phys. B 260 (1985) 1 [INSPIRE]. 
[135] L. Willmann et al., New bounds from searching for muonium to anti-muonium conversion, Phys. Rev. Lett. 82 (1999) 49 [hep-ex/9807011] [InSPIRE].

[136] J.E. Kim, P. Ko and D.-G. Lee, More on R-parity and lepton family number violating couplings from muon(ium) conversion and tau and piO decays, Phys. Rev. D 56 (1997) 100 [hep-ph/9701381] [INSPIRE].

[137] J. Kopp, L. Michaels and J. Smirnov, Loopy constraints on leptophilic dark matter and internal bremsstrahlung, JCAP 04 (2014) 022 [arXiv: 1401.6457] [INSPIRE].

[138] R. Essig et al., Working group report: new light weakly coupled particles, arXiv:1311.0029 [INSPIRE].

[139] J. Alexander et al., Dark sectors 2016 workshop: community report, arXiv:1608.08632 [INSPIRE].

[140] J.D. Bjorken, R. Essig, P. Schuster and N. Toro, New fixed-target experiments to search for dark gauge forces, Phys. Rev. D 80 (2009) 075018 [arXiv:0906.0580] [INSPIRE].

[141] S. Gardner, R.J. Holt and A.S. Tadepalli, New prospects in fixed target searches for dark forces with the Seaquest experiment at Fermilab, Phys. Rev. D 93 (2016) 115015 [arXiv: 1509.00050] [INSPIRE].

[142] A. Dery, A. Efrati, Y. Nir, Y. Soreq and V. Susič, Model building for flavor changing Higgs couplings, Phys. Rev. D 90 (2014) 115022 [arXiv:1408.1371] [INSPIRE].

[143] S. Baek and Z.-F. Kang, Naturally large radiative lepton flavor violating Higgs decay mediated by lepton-flavored dark matter, JHEP 03 (2016) 106 [arXiv:1510.00100] [INSPIRE].

[144] X. Liu, L. Bian, X.-Q. Li and J. Shu, Type-III two Higgs doublet model plus a pseudoscalar confronted with $h \rightarrow \mu \tau$, muon $g-2$ and dark matter, Nucl. Phys. B 909 (2016) 507 [arXiv: 1508.05716] [INSPIRE].

[145] CMS collaboration, Search for lepton flavour violating decays of the Higgs boson in the $\mu-\tau$ final state at $13 \mathrm{TeV}$, CMS-PAS-HIG-16-005 (2016).

[146] ATLAS collaboration, Search for lepton-flavour-violating decays of the Higgs and $Z$ bosons with the ATLAS detector, Eur. Phys. J. C 77 (2017) 70 [arXiv:1604.07730] [inSPIRE].

[147] I. Galon and J. Zupan, Dark sectors and enhanced $h \rightarrow \tau \mu$ transitions, arXiv:1701.08767.

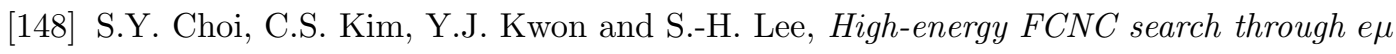
colliders, Phys. Rev. D 57 (1998) 7023 [hep-ph/9707483] [INSPIRE].

[149] BeLLE collaboration, I. Jaegle, Search for the dark photon and the dark Higgs boson at Belle, Phys. Rev. Lett. 114 (2015) 211801 [arXiv:1502.00084] [INSPIRE]. 